This is the accepted version of the following article:

Quesada, D. and Merkoçi, A. Nanoparticle-based lateral flow biosensor in Biosensors and bioelectronics (Ed. Elsevier), vol. 73 (Nov. 2015), p. 47-63.

Which has been published in final form at DOI 10.1016/j.bios.2015.05.050.

(C) 2015. This manuscript version is made available under the CC-BYNC-ND 4.0 license http://creativecommons.org/licenses/by-nc$\underline{\mathrm{nd} / 4.0 /}$ 
Graphical Abstract

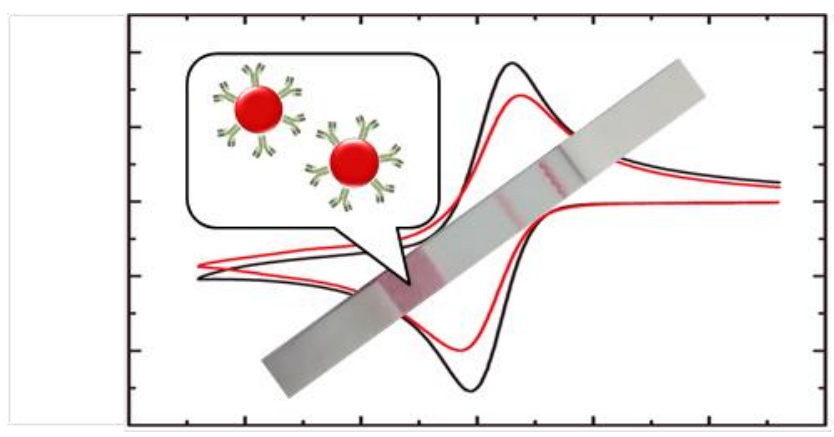




\title{
Nanoparticle-based lateral flow biosensors
}

\author{
Daniel Quesada-González ${ }^{\mathrm{a}}$ and Arben Merkoçi ${ }^{\mathrm{a}} \mathrm{b}_{*}$ \\ ${ }^{a}$ Nanobioelectronics \& Biosensors Group, Institut Català de Nanociència i Nanotecnologia (ICN2), Campus UAB, 08193 \\ Bellaterra, Barcelona, Spain \\ ${ }^{b}$ ICREA, Institució Catalana de Recerca i Estudis Avançats, Barcelona 08010, Spain. \\ *arben.merkoci@icn.cat
}

\begin{abstract}
Lateral flow biosensors (LFBs) are paper-based devices which permit the performance of low-cost and fast diagnostics with good robustness, specificity, sensitivity and low limits of detection. The use of nanoparticles (NPs) as labels play an important role on the design and fabrication of a lateral flow strip (LFS). The choice of NPs and the corresponding detection method directly affect the performance of these devices. This review discusses aspects related to the application of different nanomaterials (e.g. gold nanoparticles, carbon nanotubes, quantum dots, up-converting phosphor technologies, and latex beads, between others) in LFBs. Moreover, different detection methods (colorimetric, fluorescent, electrochemical, magnetic, etc.) and signal enhancement strategies (affording secondary reactions or modifying the architecture of the LFS) as well as the use of devices such as smartphones to mediate the response of LFSs will be analysed.
\end{abstract}




\section{Contents}

1. Introduction

1.1. Lateral flow biosensors (LFBs)

1.2. How lateral flow strips (LFS) work?

2. Optical detection

2.1. Gold nanoparticles (AuNPs)

2.1.1. Design and applications of LFBs with AuNPs

2.1.2. Enhancement strategies

2.1.2.1. Modification of the labels

2.1.2.2. Architecture tuning

2.2. Fluorescent nanoparticles

2.2.1. Quantum dots (QDs)

2.2.2. Other fluorescent materials

2.3. Other nanoparticles

2.3.1. Carbon based materials

2.3.2. Colored nanoparticles

2.3.3. Dyed beads and liposomes

3. Electrochemical detection

4. Other detections

4.1. Magnetic methods

4.2. Other methods

5. Integration and connection with real world applications

6. Conclusions

Acknowledgements

References

Abbreviations 


\section{Introduction}

Nowadays biosensors are very helpful tools in our everyday life, being used for the detection of allergens in food, toxicants in water, in chronic diseases control, pregnancy tests and other diagnostic applications. Certainly, it can be ensured that biosensors are going to enter even deeper in our life in the future, so it is a research field looking for new and improved easy-to-be-used device technologies.

a)

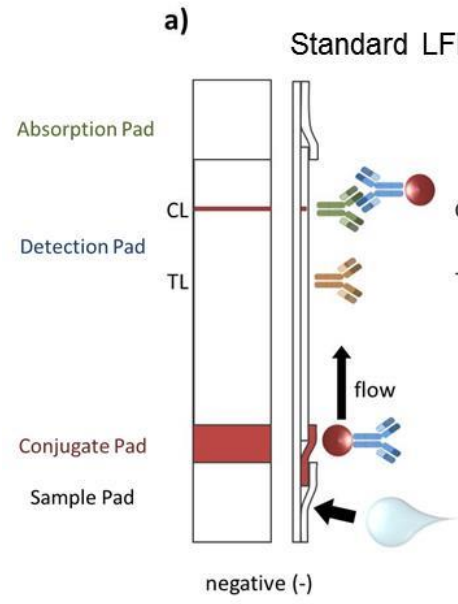

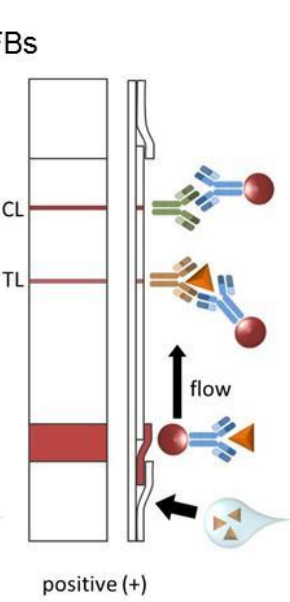

b)

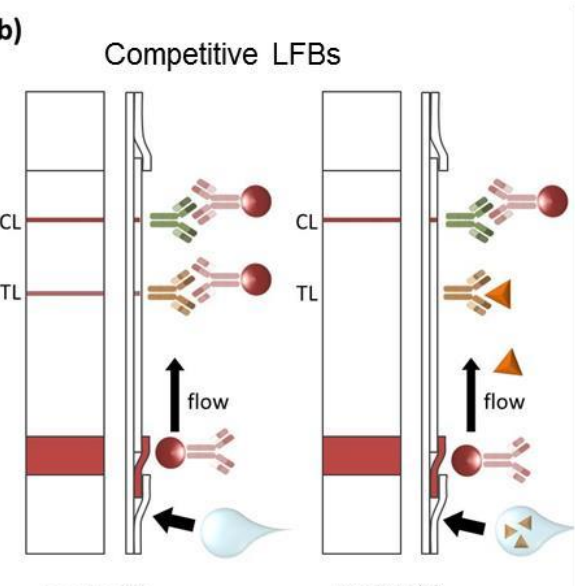

negative (-)

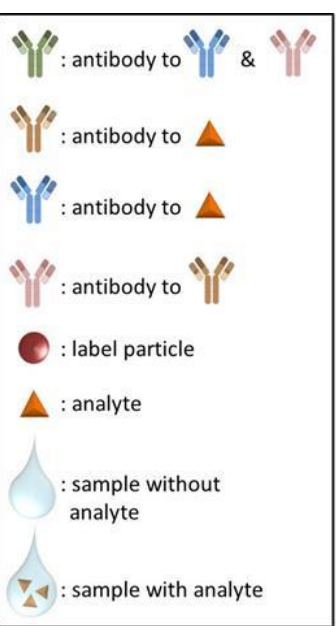

Fig. 1. Schematic representation of the different parts of a LFS (lateral flow strip) and movement of analytes and label particles across it with a) standard and b) competitive designs.

Since the appearance of the first biosensor (Clark et al., 1953), the technology has evolved, but it is still not crowned with the expected devices that would work as easily as a glucose biosensor or a pregnancy test, present in any pharmacy all over the world. Low cost and efficient devices for the detection of other analytes as DNA, proteins or even whole cells in real scenarios are still in the way.

One of the possible paths that the researchers could take to reach this future is the development of paper-based biosensors, following the same principle as the immunochromatographic assays (IAs) (Lou et al., 1993; Cho and Paek, 2001; Lönnberg and Carlsson, 2001; Ho and Wauchope, 2002; Shyu et al., 2002): the separation of analytes which flow across a porous medium taking into account the specific interactions that occur between antigen and antibody, enzyme and substrate, or receptor and ligand. Paper is a simple, cheap, abundant and an easy-to-manufacture material that fulfils cost/efficient requirements in biosensing technology (Costa et al., 2014). It is noteworthy that it is in developing countries where this type of biosensors are more requested due to the lack of resources to use conventional laboratory tools which are more expensive and require trained operators, huge amount of equipment and installations, so the development of paper-based devices could be of vital importance in these regions.

Paper, this mere material made from cellulose (the most abundant polymer on Earth) or nitrocellulose, offers many others advantages in the development of biosensors. Various biochemical reactions with interest for biosensing applications can easily be carried out within this matrix. In addition, simple microfluidics including platform architectures tuning can be applied thanks to the controlled porosity and capillary forces of the nitrocellulose network in addition to simple modification or integration processes. Moreover paper-based platforms are compatible with either naked eye detection or simple optical or electrical readers.

\subsection{Lateral flow biosensors ( $L F B$ s)}

The aim of this review is to discuss and analyze the current advances concerning the class of paper-based biosensors, called lateral flow biosensors (LFBs), which are the modern version of paper IAs (Parolo and Merkoçi, 2013). These devices may fit all the requirements expected from a biosensor: low limit of detection, high sensitivity, good selectivity, low quantity of sample volume required, no washing steps are necessary, robustness, low cost, quick assay performance in just one step and a user-friendly format. Nevertheless LFBs also have some weaknesses, such as the fact that the response obtained on naked-eye is just qualitative, not quantitative, although with the help of certain reading devices it can be converted into semiquantitative. Another drawback is that the sample must be always in liquid state, with enough viscosity to flow across the porous of the nitrocellulose. These pores, in some cases, could be obstructed by different matrix compounds and provoke unspecific adsorptions in the membrane; it is in those cases when a sample pretreatment or predilution will be required. Because of the limitation of the detection area, the surface where receptors (e.g. antibodies, enzymes, proteins, etc.) are placed, at higher concentrations of analyte, can be oversaturated, giving false blank response; it is another factor to consider making the predilution of the sample before the analysis.

LFBs can be used to detect a large range of biomarkers that may include not only proteins but also nucleic acids and even whole cells, among other biocompounds. Furthermore, LFBs are not limited only to biomolecules detection; several publications have appeared in the last years about the detection of pollutants such as metallic ions, pesticides, etc. The range of LFBs applications is 
including detection of hazardous substances (Shyu et al., 2002), heavy metals in drinking waters (Mazumdar et al., 2010; Torabi and Lu, 2011; Kuang et al., 2013; López-Marzo et al., 2013a; 2013b), allergens and pathogens in food (Wang et al., 2007; Shukla et al., 2011; Preechakasedkit et al., 2012; Leem et al., 2014; Berlina et al., 2013; Anfossi et al. 2013), pesticides (Zhou et al., 2004; Kim et al., 2011; Wang et al., 2014a), drugs screening (Inoue et al., 2007), etc.

There exist different commercial LFBs (Cazacu et al., 2004; Held et al., 2013), being pregnancy and fertility tests the most known examples beside tests for HIV (Pesce et al., 2006), drugs of abuse, Malaria (Cordray et al., 2012; He et al., 2014; Kersting et al., 2014), etc. Behind LFBs there is a well-known technology (Qian and Bau, 2003; Qian and Bau, 2004; Assadollahi et al., 2009; Lee et al., 2012; Linares et al., 2012) with several publications reporting different modifications of the standard designs/structure, either in terms of the materials used as transducers for the signal generation (Linares et al., 2012), in the methodology employed to translate the signal or in improving the device with different enhancement strategies.

With the recent development and explorations of nanomaterials in the field of sensors and biosensors LFB has been taking advantages for their use as alternative materials to improve their performance requested in real sample applications. Application of nanomaterials in DNA, protein, cell and various inorganic/organic compounds in various biosensing technologies is now being extended to LFB field bringing interesting results to this technology (Walcarius et al., 2013; Merkoçi, 2010; de la Escosura-Muñiz et al.; 2010, Parolo et al., 2013a; Perfezou et al., 2012; Aragay et al., 2011, 2012).

\subsection{How lateral flow strips (LFS) work?}

LFBs are manufactured in strip form, a convenient format for the user, normally with a width between 4-6 mm and a length no more than 6-7 cm. A standard lateral flow strip (LFS) consists of four main sections made of different materials, as shown in Fig. 1: sample pad, made of cellulose, where the sample is dropped; conjugate pad, made of glass fiber, impregnated with the bioconjugates solution (the label particle and a receptor for the analyte); detection pad, a nitrocellulose sheet (Lee et al., 2012; Ahmad et al., 2009) where test line (TL) and control line (CL) are printed; and absorption pad, also made of cellulose. Other additional parts can be integrated on LFS as blood filters, substituting the sample pad, to retain big particles like blood red cells and avoiding their hemolysis. Another example of material which can be integrated on LFS is carbon nanotubes paper, with high conductive properties to connect LFS to electronic devices (Zhu et al., 2014).

The performance of an assay with a LFS is quite simple: the sample is added on the sample pad and then the liquid will start flowing to the conjugate pad where the analyte, if present on the sample, will be linked to the transducers (the label particles), previously conjugated with a bioreceptor specific to the analyte. The conjugate, rehydrated by the liquid, will flow by capillarity forces across the detection pad to the absorbent pad, passing through the TL, where it will be captured only if the conjugate has the analyte attached (positive response), and to the CL, being always captured, evidencing that the assay works. This design corresponds to a standard model of LFS (Fig. 1a), but there exists also the competitive model (Fig. 1b) in which the analyte and the transducers compete for being captured on TL, obtaining a response inversely proportional to the concentration of analyte.

\section{Optical detection}

Although a large variety of strategies to read the LFSs signal has been reported the optical methods remain the most explored because of their simplicity (even naked eye detection) and effectiveness (high sensitivity). Several types of nanomaterials can be used as colorimetric labels on LFBs conjugated with different kinds of bioreceptors (antibodies, aptamers, DNA etc.). In the following sections we will revise the most relevant technological and analytical aspects related to the application of LFS labels such as gold nanoparticles (AuNPs), quantum dots (QDs), carbon nanotubes (CNTs), carbon nanoparticles (CNTs) and liposomes between others.

\subsection{Gold nanoparticles (AuNPs)}

AuNPs are easy to synthesize and manipulate, stable in time, size-tunable, biocompatible and have an intense red color easy to be detected even by naked eye or usually using color readers to achieve better detection limits. Because of these properties, AuNPs are the most reported nanomaterial used as optical label in LFSs. 


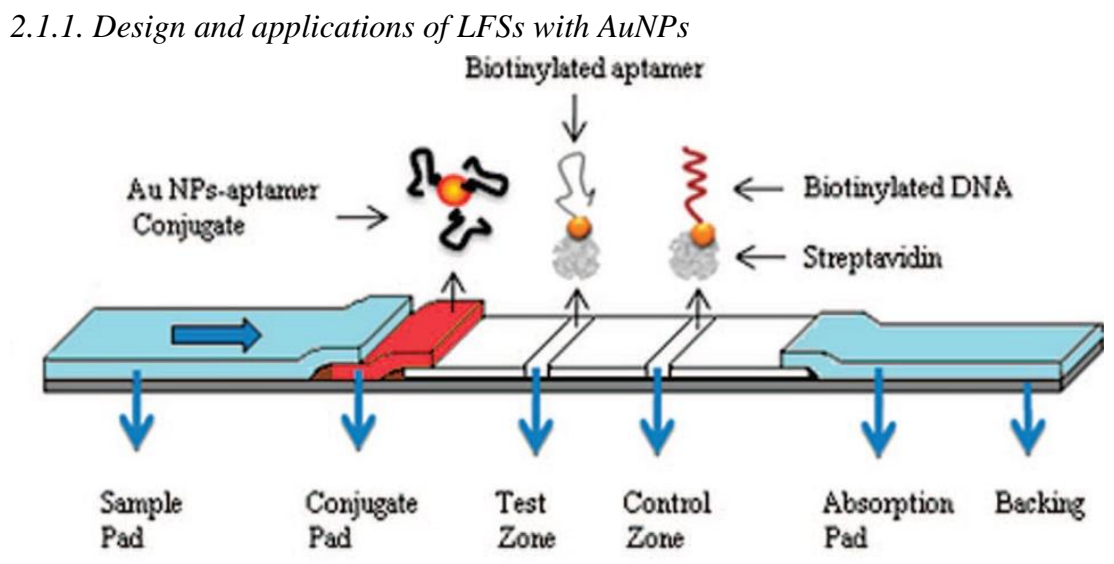

Fig. 2. LFS with aptamers conjugated AuNPs. Reprinted with permission from Xu, H.; Mao, X.; Zeng, Q.; Wang, S.; Kawde, A. N.; Liu, G., 2009, Anal. Chem., 81, 669-675. Copyright 2009 American Chemical Society.

One of the first examples of the use of AuNPs as labels on LFSs was reported by Shyu et al. (2002) for the detection of ricin. On this LFS, without conjugate pad neither sample pad, anti-ricin antibody conjugated AuNPs were deposited over nitrocellulose membrane near the bottom of the strip.

Few years later, the number of reports about LFBs increased and the design evolved including a pad, made of glass fiber, for the AuNPs suspension (Oh et al., 2006). Also, besides antibodies, other biocompounds like aptamers (Liu et al., 2006; Liu et al., 2009; Xu et al., 2009) and DNA probes (Mao et al., 2009; He et al., 2012; Rohrman et al., 2012; Lie et al., 2012; Kolm et al., 2015) were conjugated with AuNPs. Xu et al. (2009) designed a LFS with AuNP-aptamer conjugate as label (Fig. 2) for the detection of thrombin. The system demonstrates that the use of the thrombin aptamer exhibits a high sensitivity, comparable or even superior to the systems which use antibodies. The experiment shows that the color intensity of AuNPs can be detected up to a concentration of analyte of 0.6 pM in diluted plasma samples being this value lower than in antibody-based assays (Shyu et al., 2002; Oh et al., 2006; Karakus, 2015), without detecting any unspecific adsorption; that evidences the good selectivity of aptamers combined with AuNPs.

It was also demonstrated that AuNPs can be used not only for biocompounds detection (Karakus, 2015); toxins (Anfossi et al., 2013; Liu et al., 2014) and metallic cations (Mazumdar et al., 2010; Torabi and Lu, 2011; Kuang et al., 2013; López-Marzo et al., 2013a; 2013b; Fang et al., 2010) also can be detected using several strategies. In the case of the work of Fang et al. (2010), $\mathrm{Cu}^{2+}$ ions can be detected taking advantage of the cleavage that cooper causes, in presence of ascorbate, on $\mathrm{Cu}^{2+}$-specific-DNAzymes (linked to AuNPs) which, if broken, will be captured on TL. This strategy grants a good selectivity against other metallic ions, but the limit of detection does not reach the levels achieved in the detection of antigens. An alternative high sensitive strateg y to detect metallic ions consists on the use of antibodies specific to a metal-ligand complex but not to the free metal. The metal-ligand complex competes for being hooked on the test line with the same complex but already linked to AuNPs, thereby obtaining a competitive format of LFSs. This strategy is seen on López-Marzo et al. (2013a)'s work to detect $\mathrm{Cd}^{2+}$ ions using EDTA as ligand. The system, nevertheless, is shown to have some problems of unspecificity to some metals; however it can be solved reducing the concentration of the ligand but also adding extra conjugation pad that ensures $\mathrm{Cd}^{2+}$ complexation with EDTA and interference masking through ovalbumin (OVA) (López-Marzo et al., 2013b; to be discussed in 2.1.2.2. architecture tuning section). 


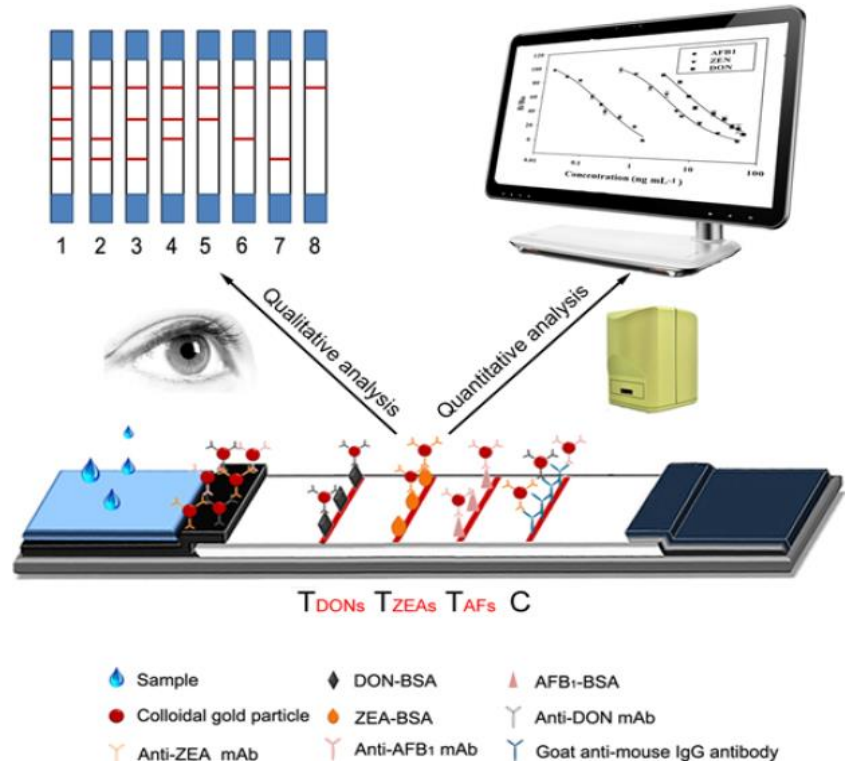

Fig. 3. LFS for the multidetection of different mycotoxins. Qualitative (left) and quantitative (right) response. Reprinted with permission from Song, S.; Liu, N.; Zhao, Z.; Ediage, E. N.; Wu, S.; Sun, C.; Saeger, S. D.; Wu, A., 2014, Anal. Chem., 86, 4995-5001. Copyright 2014 American Chemical Society.

In some cases, multidetection of analytes could be required, so it would be necessary to use various nanoparticles easy to be concentrated so as to achieve an intense color. Hereby, AuNPs are a good choice to develop multidetection LFSs (Elenis et al., 2011; Wang et al., 2013; Song et al., 2014; Kim et al., 2014). These LFSs could replace ELISA assays, which are time-consuming and expensive, as was demonstrated by the work of Song et al. (2014), developed to detect different mycotoxins at the same time (Fig. 3). Their work, additionally, demonstrates that LFSs, besides of the qualitative response, permit the quantitative interpretation of the signal by means of a colorimetric reader. The quantitative response can be represented as relative optical density (the ratio in percentage between the signal of a positive sample and the blank). This data can be used to construct a calibration curve of the measuring system.

The work of Chen et al. (2012) and Huang et al. (2014) reveals another interesting application of AuNPs on LFSs, the development of logic gates systems. AuNPs can afford more than one biocompound conjugated on their surface, therefore easily a LFS could detect the presence of various analytes on the same line ("OR" logic gate: the LFS will mark positive signaling in the presence of any of the analytes). Also the signal can indicate only the presence of both analytes at the same time (Chen et al., 2012) ("AND" logic gate: the signal on TL will only appear if both analytes are present). Huang et al. (2014)'s system can also detect the presence of a single analyte in a system where the analytes are inhibited by each other ("INH" logic gate: the LFS will mark positive signaling in the presence of only one of the analytes, but not if both are present in the sample at the same time).

\subsubsection{Enhancement strategies}

Although LFBs with AuNPs have demonstrated to have good sensitivity and low limits of detection, there are cases when it is necessary to achieve better performance. Different enhancement strategies with potential to significantly improve LFSs devices have been reported. These strategies can be based on chemical methods, like the modifications of the label's surface (the AuNPs) to afford secondary reactions or attachments, or in altering the physical properties of the device (flow movement, speed, etc.) by modifying the design/architecture. 


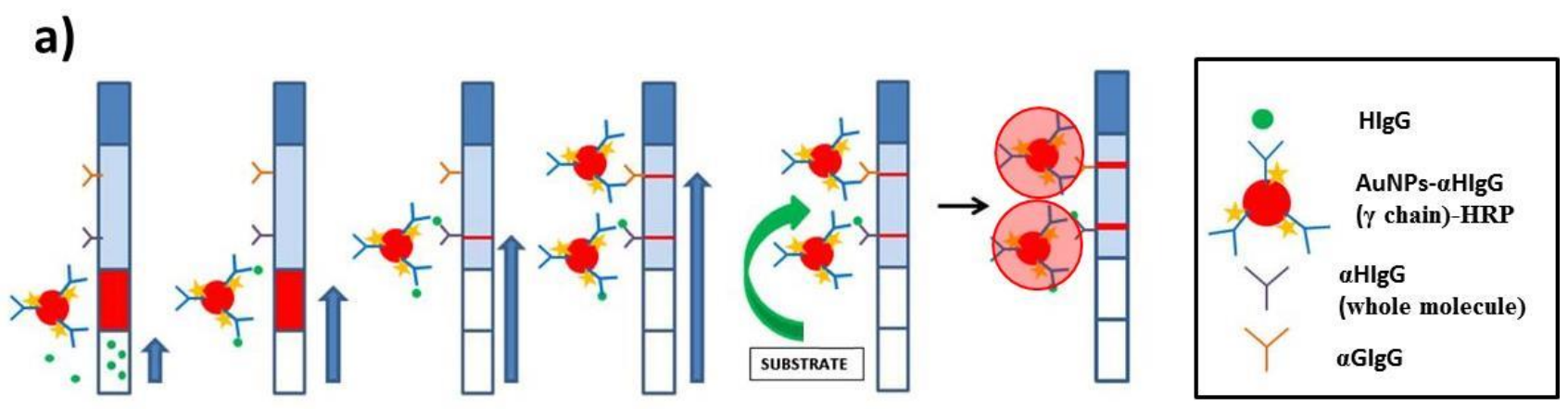

b)

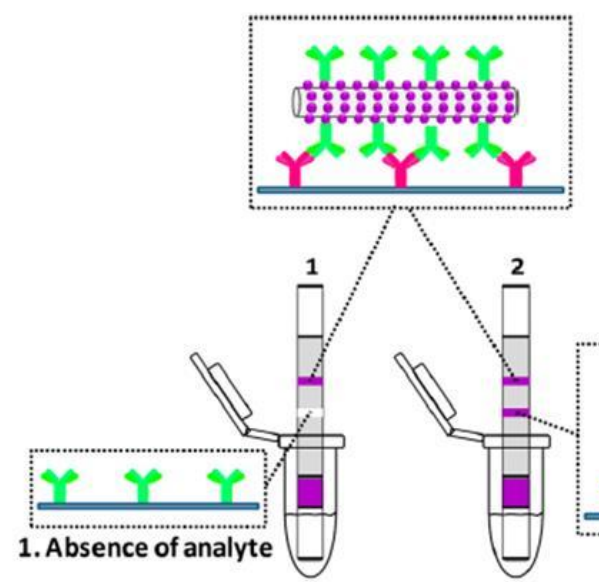

c)

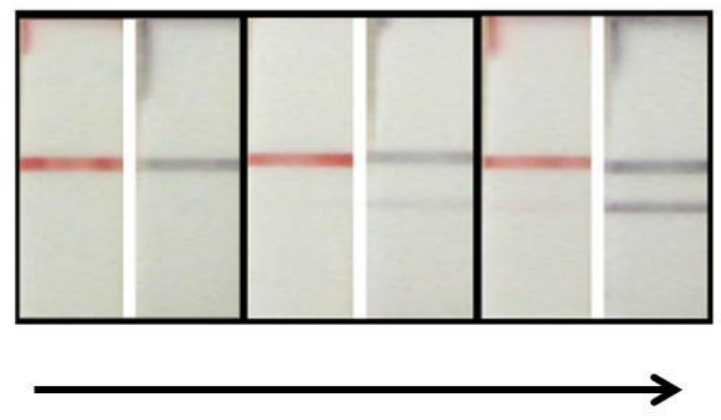

concentration of analyte: rabbit IgG

Fig. 4. a) Signal enhancement strategy on LFS by means of enzymatic reactions. Adapted with permission from Parolo, C.; de la Escosura-Muñiz, A.; Merkoçi, A., 2013b, Biosens. Bioelectron., 40, 412-416. Copyright 2013 Elsevier. b) AuNPs carried on silica nanorods: design and performance of the LFBs assay and c) comparison of results with standard AuNPs labels (left strips) and silica nanorods decorated with AuNPs (right strips). Adapted with permission from Xu, H.; Chen, J.; Birrenkott, J.; Zhao, J. X.; Takalkar, S.; Baryeh, K.; Liu, G., 2014, Anal. Chem., 86, 7351-7359. Copyright 2014 American Chemical Society.

\subsubsection{Modification of the labels}

One of the strategies used to amplify the signal on TL and CL is to surround AuNPs with other compounds which enhance the color by means of enzymatic reactions (Fig. 4a), as the reaction occurring between HRP (horseradish peroxidase) and some chromogenic substrates (Mao et al., 2009; He et al., 2011; Parolo et al., 2013b), such as AEC (3-amino-9-ethylcarbazole) or TMB (3,3',5,5'-tetramethylbenzidine). AEC mixed with $\mathrm{H}_{2} \mathrm{O}_{2}$, use to provide color enhancement (and consequently, higher sensitivity) on HRP catalyzed reactions. Nevertheless it is when using TMB that the quantification limit is further decreased due to the fact that this substrate grants higher contrast between the lines and the background than the AEC. The reported detection limit of these enhancement strategies can reach the $200 \mathrm{pg} / \mathrm{mL}$ (Parolo et al., 2013b).

Although enzymatic reactions exhibit low limit of detection and good sensitivity, the short stability of the reagents and the long conjugate development and testing times are drawbacks to consider. The amplification method proposed by Rastogi et al. (2012) by means of gold deposition over the strip after the assay solves the problem of reagents stability and seems to increase the signal effectively at low concentrations, but at higher concentrations, when detection lines are already saturated, the signal enhancement is slight. Fridley et al. (2012) studied the release of dry reagents stored in porous media (rehydrated afterwards) and demonstrated that this strategy could be used to enhance up to four times the intensity of AuNPs (previously deposited on the strip). This strategy, if developed on a real assay (with AuNPs and analyte flowing) could lessen the testing time in comparison with the methods in which amplification is performed after the assay. However, it is important to be taken into account the properties of the reagents to be stored on the membrane, their stability once dried and their potential as signal enhancers after rehydration. 
Another interesting work was reported by Li et al. (2012). Taking advantage of the quenching effect of gold ions on QDs, they dissolved the AuNPs captured on TL using $\mathrm{HCl}-\mathrm{Br}_{2}$ mixed solution and, in a 96-well microplate, they measured the fluorescence of QDs. The method provides a good sensitivity, a wide working range and a fairly low limit of detection $(90 \mathrm{pg} / \mathrm{mL})$. Despite these advantages, the procedure is long and difficult to execute on field since it requires treating of the strips, fluorescence equipment to read the 96-well microplate and trained personnel. Instead of solving the AuNPs, Shi et al. (2015a) developed a system which allowed fluorescence quenching measurements without the need of dissolving the nanoparticles; they dispensed fluorescent polymer dots of 50nm on the TL and CL and observed how the fluorescence decreased with the increase of concentration of AuNPs, which competed against the analyte. The detection limit of their method reached the $160 \mathrm{pg} / \mathrm{mL}$. As an alternative to fluorescence detection, silver deposition onto AuNPs could be used to enhance the colorimetric response, turning into black the color of TL and CL (Anfossi et al., 2013). This strategy not necessary requires the use of colorimetric reader, if only qualitative assay is needed, but the detection limits achieved are not as low as when using QDs and fluorescence.

Recently, Xu et al., 2014, developed LFS in which, as labels, AuNPs were loaded over silica nanorods surface (Fig. 4b), raising quite a lot the sensitivity of the method due to the increase of AuNPs density over detection lines (Fig. 4c). The detection limit also is improved, in comparison to the previous mentioned strategies, being $10 \mathrm{pg} / \mathrm{mL}$ the estimated value. Another observable improvement of this method is that the amount of antibodies dispensed on TL and conjugated onto AuNPs could be reduced. Nevertheless, one should keep in mind that the silica nanorods preparation and their decoration with AuNPs could be a timeconsuming step, besides that the size of the rods (tens of micrometers) slows the flow across the membrane thus increasing the testing time. The size of the labels also precludes the use of small pore membranes, which use to exhibit higher sensitivity than the membranes with bigger pores.

a)

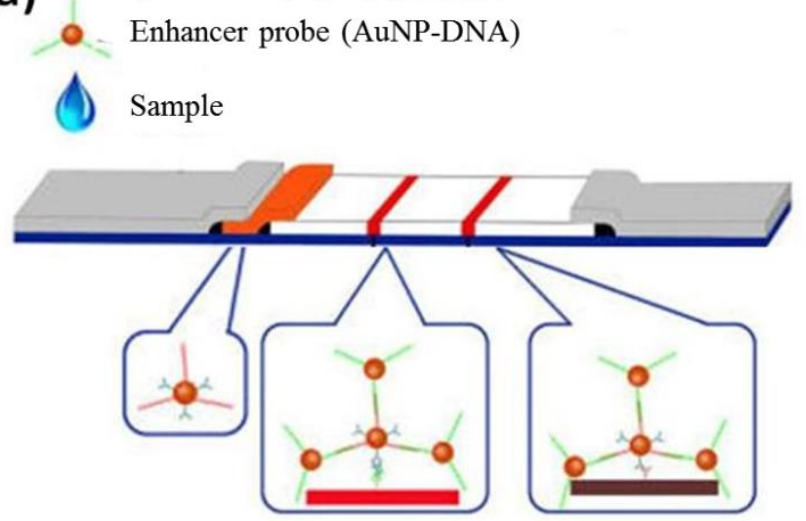

b)

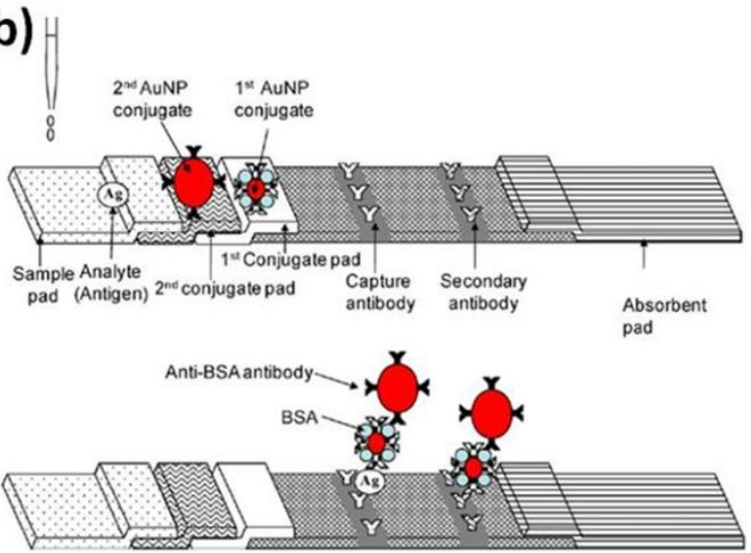

Fig. 5. Strategies to increase the density of AuNPs on TL and CL: a) LFS with AuNPs-probes cocktail on the conjugate pad. Adapted with permission from Ge, C.; Yu, L.; Fang, Z.; Zeng, L., 2013, Anal. Chem., 85, 9343-9349. Copyright 2013 American Chemical Society. b) LFS with double conjugate pad. Reprinted with permission from Choi, D. H.; Lee, S. K.; Oh, Y. K.; Bae, W. B.; Lee, S. D.; Kim, S.; Shin, Y. B.; Kim, M. G., 2010, Biosens, Bioelectron., 25, 1999-2002. Copyright 2010 Elsevier.

To increase the density of AuNPs on the detection lines, another strategy is to interconnect AuNPs by means of DNA probes (Hu et al., 2013; Ge et al., 2013) conjugated onto their surface. The binding between AuNPs can be performed previously to the assay, adding the AuNPs-probes cocktail to the conjugate pad (Hu et al., 2013), or during the assay, adding the enhancer nanoparticles mixed with the sample (Ge et al., 2013) (Fig. 5a). As in the case of AuNPs decorated silica rods and the deposition of dry reagents on the membrane method, these designs are user-friendly, being able to be performed in one only step, without the need for post-treatments on the strip. 


\subsubsection{Architecture tuning}

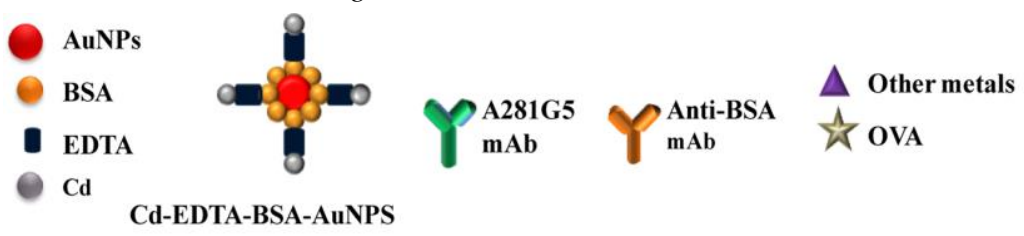

A
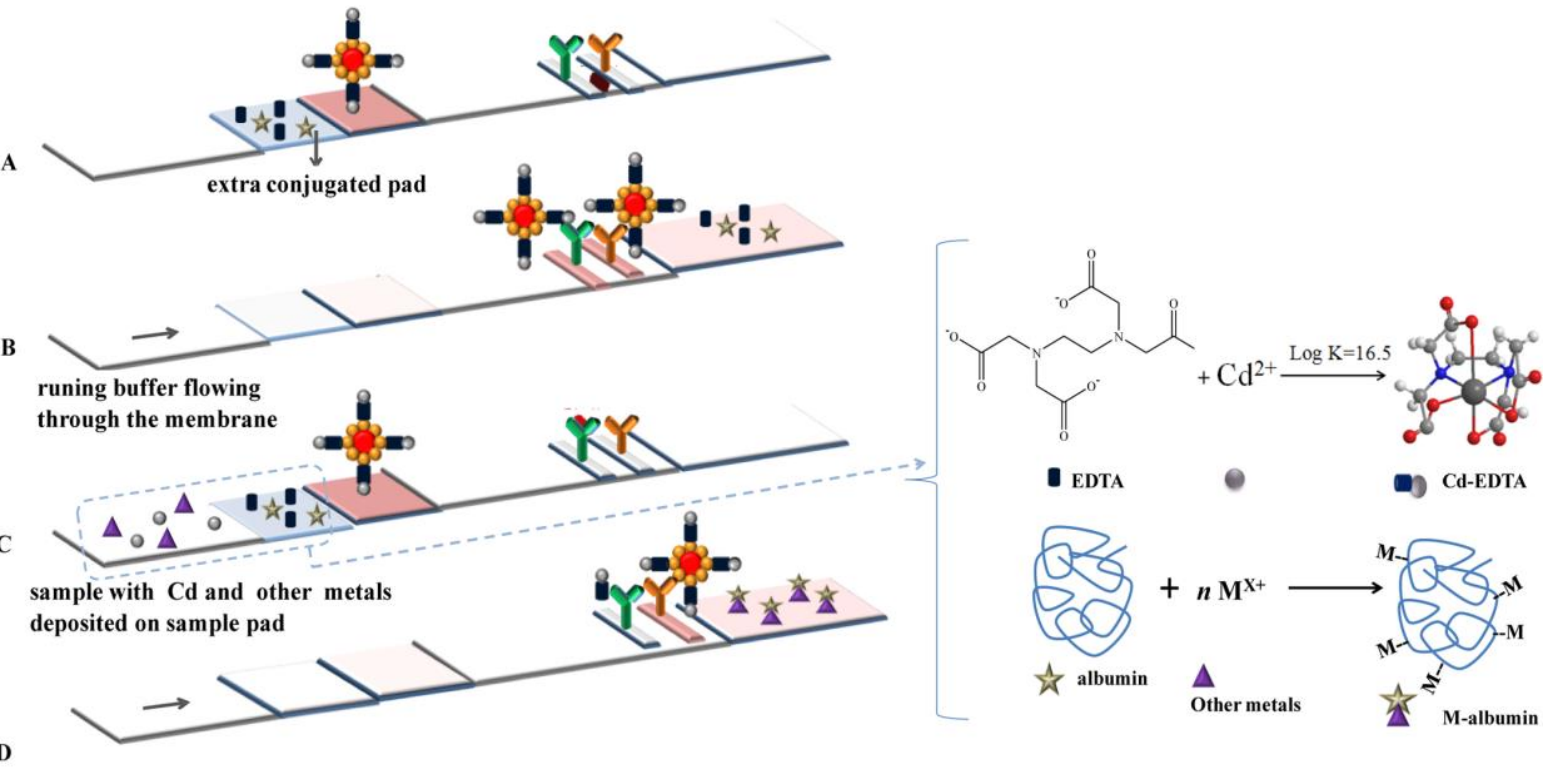

sample with $\mathrm{Cd}$ and other metals

flowing through the membrane

Fig. 6. LFS with additional conjugate pad for sample pre-treatment. Reprinted with permission from López-Marzo, A. M.; Pons, J.; Blake, A. D.; Merkoçi, A., 2013b, Anal. Chem., 85, 3532-3538. Copyright 2013 American Chemical Society.

As mentioned, increasing of the density of AuNPs on TL and CL is a good strategy to enhance the LFS signal. The LFS design modification, can be easily achieved, for example by adding an extra conjugate pad, as shown in the work of Choi et al. (2010). On this design, two different sized AuNPs conjugated with different types of antibodies on two pads are used. The smallest AuNPs are conjugated with the antibodies which are selective to the antigen and will flow faster than the biggest AuNPs, which are conjugated with anti-BSA antibodies and will bind to the previous AuNPs, covered with BSA as blocking agent, obtaining an enhanced signal due to this new sandwich format (Fig. 5b). The detection limit of this LFS is $1 \mathrm{ng} / \mathrm{mL}$, so the enhancement is not as good as in the previously discussed methods. Nevertheless this method is advantageous due to the fact that the assay is performed as a real onestep procedure, without pre-treatments on the sample neither post-treatments on the strip. Should also be noted that the preparation of both conjugates is simple and the biocompounds which are used are not very expensive. This technique was used again by Mei et al., 2013, demonstrating that it works as well for the detection of small molecules.

In cases where pre-treatment of the sample is required an extra pad can also be used to pre-treat the sample, simplifying the test procedure. In the work of López-Marzo et al. (2013b), an improvement of a previous design (López-Marzo et al., 2013a) by adding an extra pad with EDTA, which binds the metallic ions to allow their detection, and OVA, to help masking the interferences has been carried out (Fig. 6). More pads could be added, to enrich the flowing liquids with buffers or to improve the signal with enhancer solutions once the assay is finished, but it could require several steps, having to wait for the timely moment of each one, hindering the reproducibility of the device. That problem can be solved using the two-dimensional paper network format of Fu et al., (2011a). Buffer, sample and signal enhancer are added at the beginning of the assay, then the device is closed and, when the pads contact, the flowing across the paper network starts. In this manner the reproducibility of the liquid mixing is better controlled. In another work of Fu et al. (2012) they applied their knowledge about the behavior of the flow on paper-based materials (Fu et al., 2010; Fu et al., 2011b) to simplify their previous design. The possibilities are huge and of course this strategy can be adapted to many other designs (Kim et al., 2012) and transducers, not only to AuNPs (Cho et al., 2006; Liu et al., 2009; Jahanshahi-Anbuhi et al., 2012). 
a)

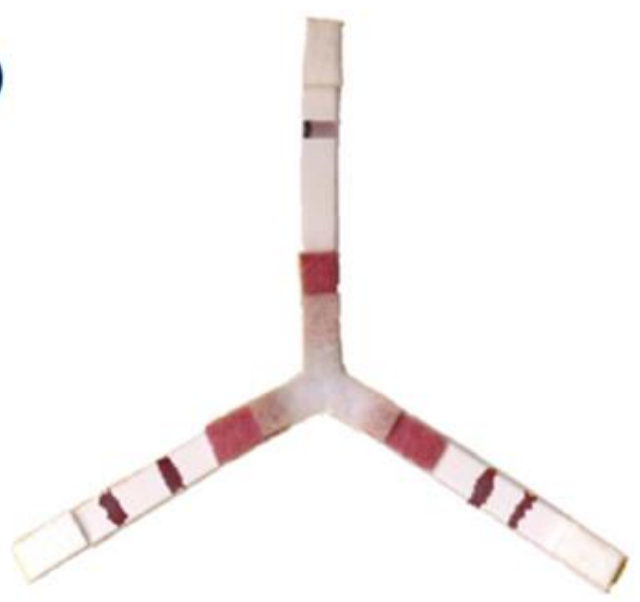

b)
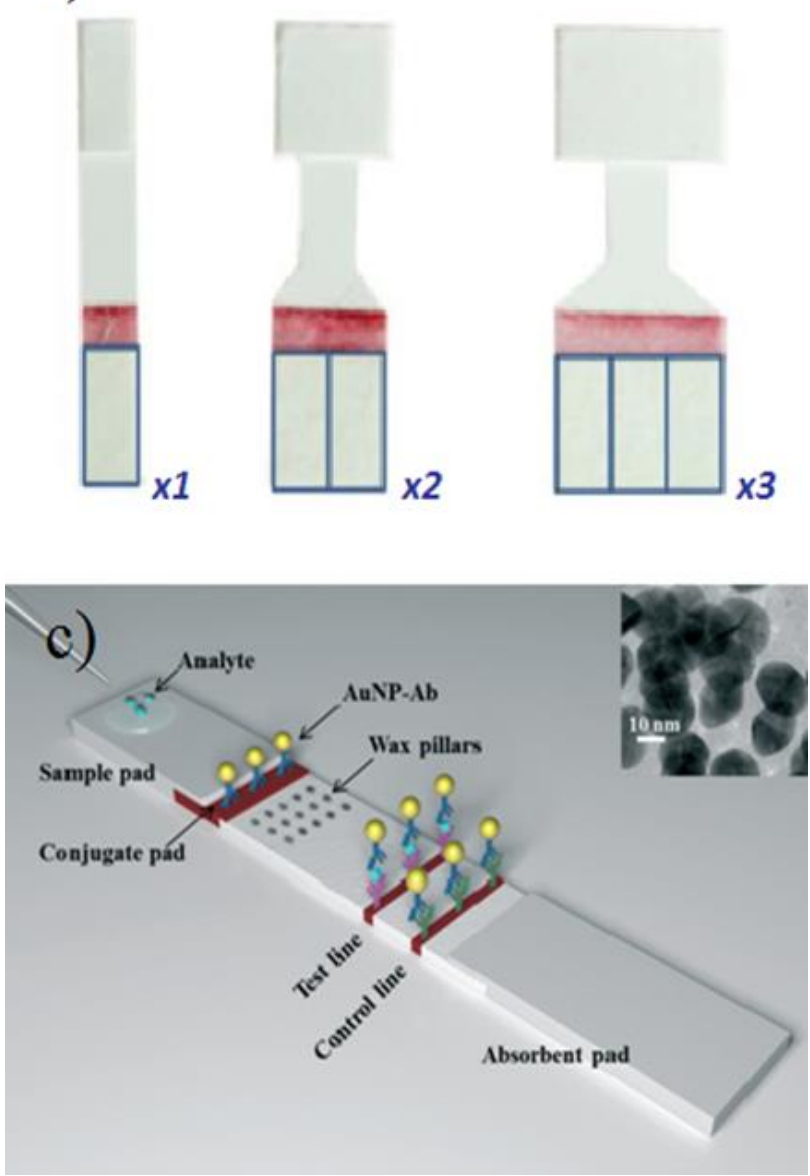

Fig. 7. Alternative LFS designs: a) Multidetection device composed by three LFS specific to different analytes. Reprinted with permission from Li, C.; Vandenberg, K.; Prabhulkar, S.; Zhu, X.; Schneper, L.; Methee, K.; Rosser, C. J.; Almeide, E., 2011, Biosens. Bioelectron., 26, 4342-4348. Copyright 2011 Elsevier. b) Paper architecture modification to enhance the signaling. Reprinted with permission from Parolo, C.; Medina-Sánchez, M.; de la Escosura-Muñiz, A.; Merkoçi, A.; 2013c, Lab Chip, 13, 386-390. Copyright 2013 Royal Society of Chemistry. c) Design with wax pillars printed on LFS membrane, to modify the flow across the pores. Reprinted with permission from Rivas, L.; Medina-Sánchez, M.; de la Escosura-Muñiz, A.; Merkoçi, A., 2014, Lab Chip, 14, 4406-4414. Copyright 2014 Royal Society of Chemistry. 
Multidetection of analytes is another interesting area in which architecture tuning can be helpful. Fu et al. (2010) and Fenton et al. (2008) experimented with the division of the flow on paper format, proving that multiplex design on lateral flow could work without the need of pumps or other auxiliary devices. Li et al. (2011) adapted LFS to the multiplex format in which three strips shared one sample pad (Fig.7a).

At the beginning of this section it was discussed how increasing the quantity of gold nanoparticles in TL and CL boosts the sensitivity of the device. In the work of Parolo et al. (2013c) this purpose was reached modifying the architecture of paper. The authors tried different designs in which the flow was accelerated inside the detection pad due to a funnel effect, which at the same time was concentrating the amount of AuNPs and analytes inside (Fig.7b). The optimal results were obtained when the width of sample pad and conjugate pad was 3 times the width of the detection pad, enhancing the sensitivity and reducing about eight times the limit of detection in comparison to a standard LFS.

Another way to modify the flow is to disturb it, instead of provoking its acceleration the time that the transducers have to conjugate with analytes can be increased, ensuring that all AuNPs are conjugated and, consequently, captured on TL and CL. In the work of Hong et al. (2012) the antibodies were dispensed as patterns of parallel and zigzag dots on the detection pad, being the zigzag pattern the one which gave the highest intensities, presumably because flow slower on this design. Recently, it has been demonstrated that wax is a useful tool due its hydrophobicity to control flow on paper devices and create channels on it (Renault et al., 2014). In the work of Rivas et al. (2014), wax is used to create pillars inside the membrane (Fig.7c) slowing the movement of the fluids and amending their mixing. Furthermore, the pressure exercised when printing the wax patterns reduces the membrane's pore size, also contributing to the brake of the flow. Again, as happened in Hong et al. (2012)'s work, the zigzag design exhibits the highest response. On Rivas et al. (2014)'s work the detection limit is improved three times regarding a non-modified strip, less than on Parolo et al. (2013c)'s method; however wax pillars compose a more inexpensive design, since it does not require higher quantities of AuNPs and antibodies, in addition that wax is a cheap material.

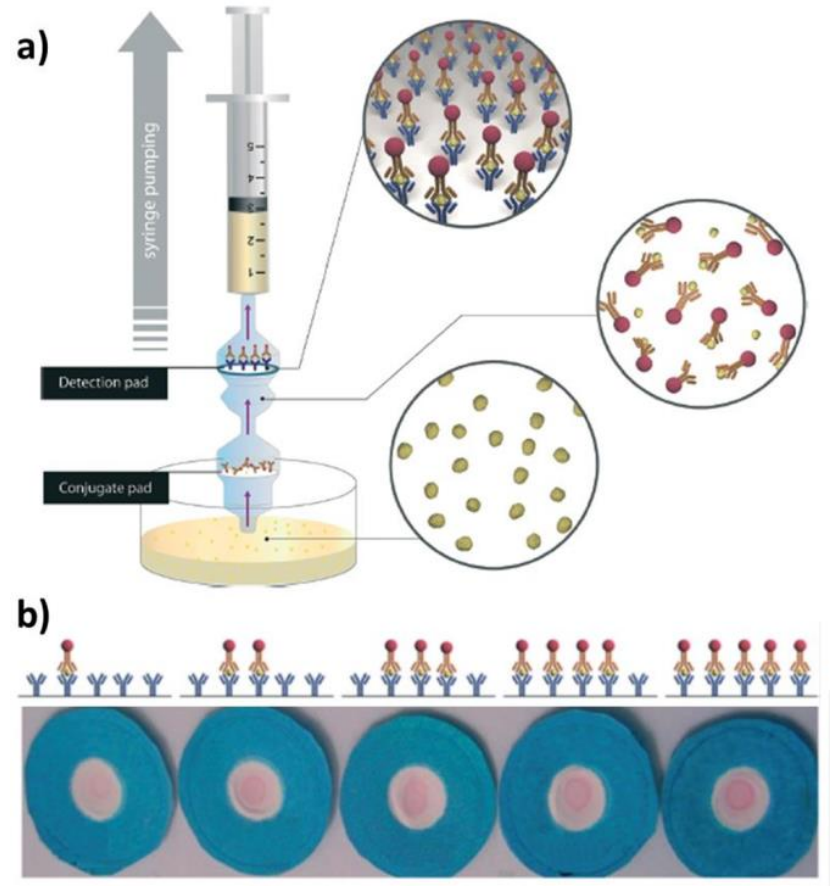

Fig. 8. Syringe design LFBs: a) Working principle b) Detection pads. Adapted with permission from Nunes Pauli, G. E.; de la Escosura-Muñiz, A.; Parolo, C.; Bechtold, I. H.; Merkoçi, A., 2015, Lab Chip, 15, 399-405. Copyright 2015 Royal Society of Chemistry.

Probably, it is the recent design of Nunes Pauli et al. (2015) the most newfangled one. Instead the use of a LFS, the assay is carried out in a vertical flow mode inside a syringe (Fig.8a); first, the sample is pumped to the conjugate pad and it interacts with it during few minutes, allowing the conjugation of the analyte with AuNPs; then, it's aspirated to the upper part (detection pad that consists in a small cartridge with circular pieces of nitrocellulose with the detection antibodies retained inside a wax ring) where the signaling takes place (Fig.8b). This technique requires high quantities of liquid in comparison to standard LFS to perform the assay; nevertheless, since the sample is being pre-concentrated in the syringe, the limit of detection is highly improved. 


\subsection{Fluorescent nanoparticles}

It is well known that fluorescence methods use to exhibit higher sensitivity that those based on absorption colorimetry. The behavior of fluorescent nanomaterials with interest to be used in LFSs has been extensively explored and some reported examples discussed below.

\subsubsection{Quantum dots $(Q D s)$}

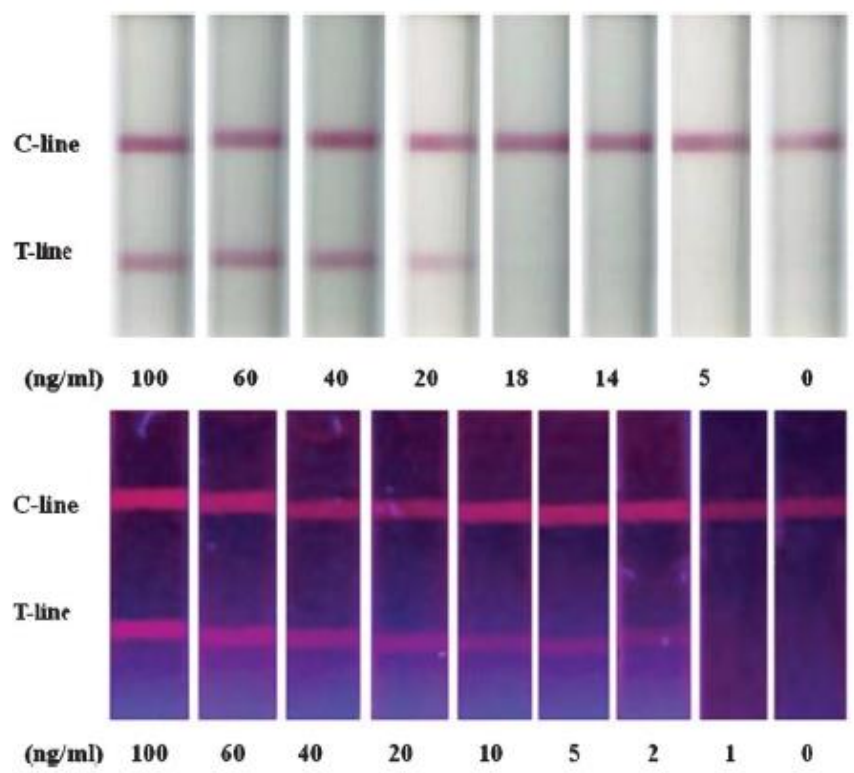

Fig. 9. Comparison of assay made with AuNPs (up) and silica nanoparticles loaded with QD (down). Reprinted with permission from Bai, Y.; Tian, C.; Wei, X.; Wang, Y.; Wang, D.; Shi, X., 2012, RSC Advances, 2, 1778-1781. Copyright 2012 Royal Society of Chemistry.

In the already discussed work of Li et al. (2012) was demonstrated that the use of quantum dots (QDs) exhibits a good sensitivity and a really low limit of detection; but on their work, quantum dots were used to indirectly detect the amount of AuNPs on TL by quenching effect. In other works QDs are used directly as labels on LFS, with both antibodies (Yang et al. 2010; Li et al., 2010; Berlina et al. 2013) and aptamers (Wang et al., 2011a; Bruno, 2014). The assays are as fast as with AuNPs, regardless the time that fluorimetric analysis could take, and the limits of detection achieved with QDs as tags are in the order of few nanograms per milliliter. The differences between an AuNPs and a QDs LFS are heighten when QDs are carried (Bai et al., 2012) or encapsulated (Ren et al., 2014) in other materials. In the work of Bai et al. (2012), QDs were loaded over silica nanoparticles, which are between 10-20 times larger than QD, and these nanocomposites were used as labels obtaining a good sensitivity and a detection limit ten times lower than with AuNPs LFS (Fig.9). These achievements were overwhelmed by Ren et al. (2014)'s work, who managed to encapsulate QDs on polymeric beads improving the limit of detection to only $0,42 \mathrm{pg} / \mathrm{mL}$ and fixing problems of flow and unspecific adsorptions of free QDs on nitrocellulose. In some cases, the conjugation of QDs with biocompounds can be a complex work due to their small size; also the number of antibodies per QDs is lower than in larger particles, so the probability of binding the label to the antigen is also a bit lower; therefore, the works of Bai et al. (2012) and Ren et al. (2014), besides enhancing the signal, they are noteworthy easing the development and behavior of the LFS.

\subsubsection{Other fluorescent materials}

Besides QDs, other fluorescent materials, like the called up-converting phosphor technologies (UPTs) reporter particles. These type of nanoparticles are considered ceramic materials of large size, few hundreds of nanometers, composed by the combination of rare earth elements, being europium one of the most used. UPT reporter particles transform low energies (infra-red) into high energies (visible light, depending on their crystalline structure). Corstjens et al. (2001, 2003, 2008a, 2008b) worked with this materials to detect nucleic acids and antigens in complex matrixes with improved results in comparison to the ELISA tests. Paterson et al. (2014) and Liu et al. (2014) also worked with this type of materials on LFSs, obtaining similar results as Corstjens et al. (2001, 2003, 2008a, 2008b). UPTs seem more stable in front of photobleaching effect (decrease of fluorescence) than QDs, making UPTs more suitable for direct assays and QDs for quenching related modes.

As seen with QDs, to carry the fluorescent tags on larger particles it helps enhancing the signal and avoids unspecific adsorptions onto the membrane. Europium, present on most of UPTs, emits fluorescence when reduced from $\mathrm{Eu}^{3+}$ to $\mathrm{Eu}^{2+}$, so several researchers have exploited that property and combined europium with microparticles (Rundström et al., 2007) and silica beads (Xia et al., 2009; Tang et al., 2015; Zhang et al., 2014) to use them as transducers of LFS. Although the results are satisfying, 
QDs and UPTs exhibit better limits of detection and working ranges. Huang et al. (2013) covered silica microparticles with $\mathrm{Ru}(\mathrm{phen})_{3}{ }^{2+}$ complex, which also exhibits fluorescent properties, obtaining a good limit of detection, $20 \mathrm{pg} / \mathrm{mL}$. Edwards et al. (2006) and Khreich et al. (2008) used liposomes to encapsulate fluorescent dyes, getting higher sensitivity when using larger particles, with limits of detection close to $20 \mathrm{pg} / \mathrm{mL}$. Commercial fluorescent microspheres which can be used on LFSs also are available (Xie et al., 2014; Wang et al., 2014b; Zhou et al., 2014). Interestingly, Xie et al. (2014) evaluated the optimal quantity of antibodies required to be conjugated with their microspheres and compared it with AuNPs, resulting that when using microspheres this quantity can be reduced four times; moreover, even using less quantity of antibodies in the labels, the sensitivity, the working range and the limit of detection are improved in comparison to AuNPs assays.

\subsection{Other nanoparticles}

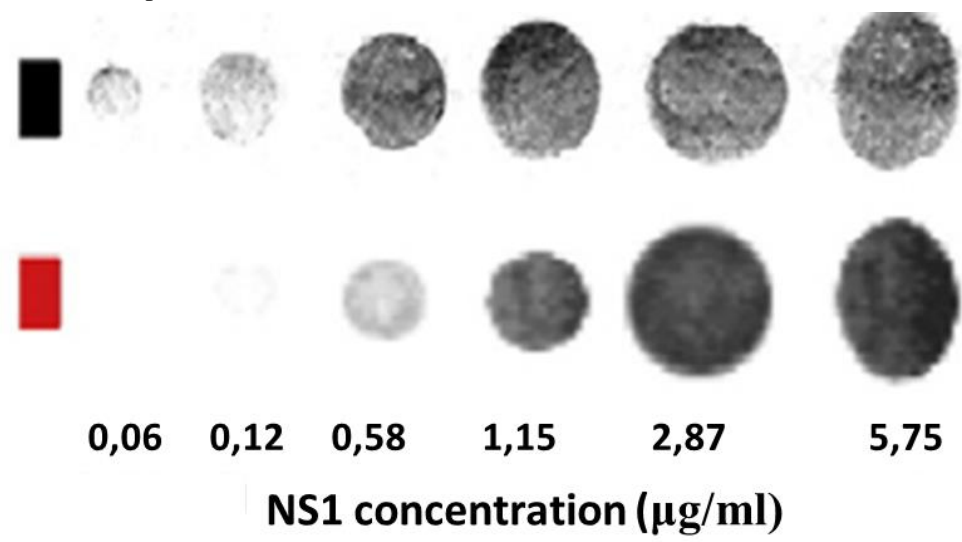

agenda

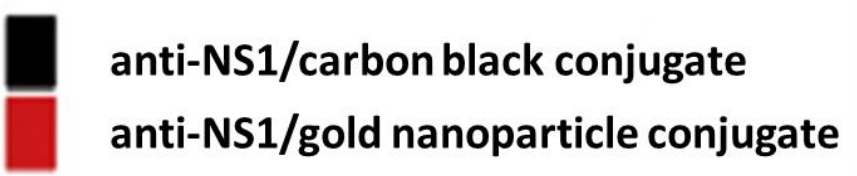

Fig. 10. Comparison of contrast obtained when using carbon nanoparticles (CNPs) and AuNPs. Reprinted with from Linares, E. M.; Kubota, L. T.; Michelis, J.; Thalhammer, S., 2012, J. Immunol. Methods, 375, 264-270. Copyright 2012 Elsevier.

\subsubsection{Carbon based materials}

Although AuNPs are the most known labels for LFSs, however other materials which, without requiring enhancement strategies or designs, have been reported to improve limits of detection. This is for example the case of carbon nanoparticles (CNPs) which in the study of Linares et al. (2012) exhibit a limit of detection ten times lower than LFS in which AuNPs or LBs (latex beads) are used. CNPs, also known as carbon black, are strongly dark colored nanoparticles that exhibit a higher contrast against the background than AuNPs (Fig.10), a factor which helps to obtain good parameters of sensitivity, working range and limit of detection. CNPs, as AuNPs, can be used for the detection of antigens (Lönnberg and Carlsson, 2001; Van Dam et al., 2004; Rayeb and Shmagel, 2008), DNA chains (Kalogianni et al., 2011; Noguera et al., 2011) and molecules (Koets et al., 2006; Blažková et al., 2009; Blažková et al., 2010), allowing also the development of multidetection LFS (Noguera et al., 2011).

Another allotropic form of carbon used on LFSs are carbon nanotubes (CNTs) (Avera and Choi, 2010; Qiu et al., 2015). CNTs are large materials, whereby using these materials on a LFSs will turn them in slow tests (around 20min to see the response), on the other hand, Qiu et al. (2015)'s work demonstrated that the limit of detection is improved in comparison to related AuNPs systems. Furthermore, CNTs are stable in time and against aggregation, which increases the life time of the conjugates.

\subsubsection{Colored nanoparticles}

One of the first nanomaterials used to develop LFBs was colloidal selenium (Lou et al., 1993), with rust color. On this firstling design, the detection of analyte was semiquantitative by means of various TL on the membrane, instead of comparing the intensity of a single TL. The detection limit of this LFS was only of few milligrams per milliliter, so rapidly selenium colloids were substituted by gold colloids which exhibit stronger color. However, several years later, Wang et al. (2014c) used again selenium nanoparticles, claiming the cost effectiveness of this material in comparison to AuNPs, to develop modern LFS. Wang et al. (2014c) could not obtain a low limit of detection, but their system with selenium nanoparticles proved to be quite specific to melamine in different matrixes.

Magnetite nanoparticles (MNPs) can be used as labels due to their strong brown color (also because of their magnetic properties, as will be discussed up ahead). Nevertheless, this strong color covers practically all the visible spectra obstructing its 
identification by means of colorimetric devices. The advantage of MNPs is that their optical properties do not change as much as AuNPs when aggregated. So Liu et al. (2011) took advantage of this property and created LFSs where MNPs were aggregated using poly-L-lysine on TL. The aggregated MNPs maintained a similar sensitivity to the non-aggregated MNPs, but the limit of detection was improved, reaching $1.7 \mathrm{ng} / \mathrm{mL}$.

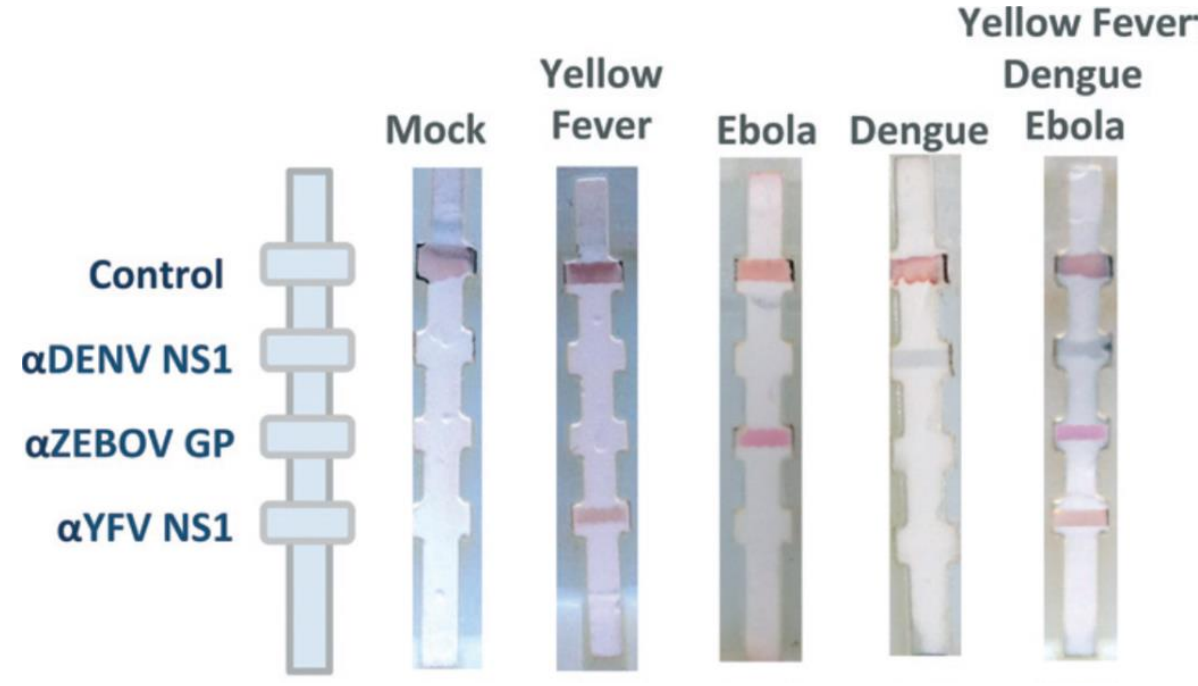

Fig. 11. Multiplex LFS developed with silver nanoparticles. Reprinted with permission from Yen, C. W.; de Puig, H.; Tam, J. O.; Gómez-Márquez, J.; Bosch, I.; Hamad-Schifferli, K.; Gehrke, Lee, 2015, Lab Chip, 15, 1638-1641. Copyright 2015 Royal Society of Chemistry.

Silver can be used to enhance the intensity of AuNPs (Anfossi et al., 2013), but silver by himself is also an interesting nanomaterial. Silver nanoparticles present different colours depending on their size and shape, so Yen et al., 2015, exploiting this property, developed multiplexed LFS in which not only each antigen had their own line, but each of the lines had a different colour, avoiding confusions to the final user (Fig.11).

In the work done by Park et al., 2015 platinum nanoparticles are used as transducers due to their properties catalysing luminol oxidation provoking chemiluminiscence. In comparison with HRP, platinum nanoparticles are more stable-in-time and robust against environmental conditions. Park et al. (2015) compared the lighting response of platinum versus the conventional AuNPs LFS obtaining a higher working range and a limit of detection one thousand times lower.

\subsubsection{Dyed beads and liposomes}

Latex beads (LBs) or polystyrene nanoparticles are homogeneous-size particles which, after being dyed, can be used to develop LFSs. Several companies use LBs on their LFSs due to the fact it is a cheap material and has a similar behavior as AuNPs. Greenwald et al. (2003) compared AuNPs and LBs and observed that LFSs prepared with LBs on conjugate pad gave a higher sensitivity and specificity than the ones prepared with AuNPs. Comparing other studies with LBs (Waters et al., 2006; Campbell et al., 2007; Lyashchenko et al., 2007), the LFSs always show a good response in terms of selectivity and sensitivity, however the limits of detection are not as low as with other materials, which makes the strips suitable for qualitative assays, for clinic tests where it is only necessary to know if an antigen is over a determinate threshold. Instead of latex, cellulose can also be used (Choi et al., 2014) as dyed label on LFSs, which is an even cheaper material.

As discussed above on Edwards et al. (2006) and Khreich et al. (2008) experiments, liposomes can be used to encapsulate dyes (whether fluorescent or not). There are some reports about the use of liposomes on LFBs (Ho and Wauchope, 2002; Baeumner et al., 2004a; Baeumner et al., 2004b; Wen et al., 2005; Edwards et al., 2006; Ho et al., 2008; Khreich et al., 2008; Shukla et al., 2011; Leem et al., 2014) with good levels of sensitivity due to the high quantities of dye that can be loaded inside the liposomes. Nevertheless, the synthesis of liposomes is often a long procedure, with difficult size-control, so the reproducibility of the test could be affected if the protocol is not well controlled. 


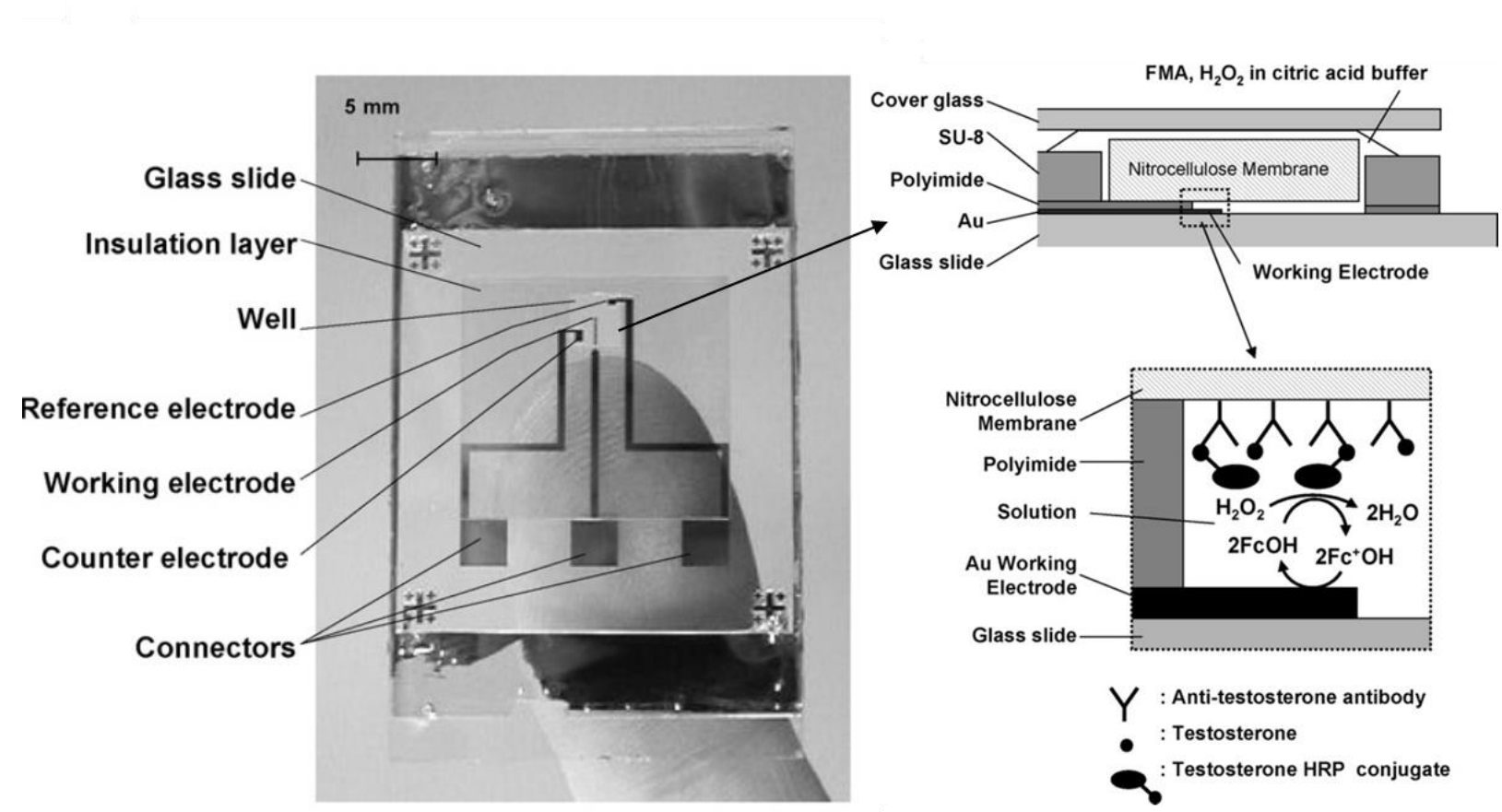

Fig. 12. Photograph of a standard electrode chip and the scheme of its well when LFS is coupled inside. Adapted with permission from Inoue, K.; Ferrante, P.; Hirano, Y.; Yasukawa, T.; Shiku, H.; Matsue, T., 2007, Talanta, 73, 886-892. Copyright 2007 Elsevier.

\section{Electrochemical detection}

Despite of the fact that LFB response can be quantitative, sometimes it can be hard to discriminate between the strips, especially by naked eye. The combination of LFBs with electrochemical detection is expected to provide a more sensitive response, higher reproducibility, wider working range and lower limits of detection than optical measurements. Nowadays electrodes can be easily miniaturized by different methods as screen printing, ink-jet printing or photolithography, allowing their easy incorporation into a LFS design. In the work of Inoue et al. (2007) the authors used photolithography to fabricate three gold electrodes, working, counter and reference (Fig. 12), over a glass slide and then coupled a small piece of nitrocellulose on a well into the device. They demonstrated that electrochemical reactions, cyclic voltammograms and amperometric measurements, could be performed on nitrocellulose substrate so, then, they cut a small piece from a LFS, corresponding to the TL, and carried out the measurements. The amperometric responses, based on the reduction of ferrocenemethanol catalysed by HRP (which was previously linked to a known quantity of testosterone, used also as analyte), decreased when the concentration of the analyte was increased in the sample (competitive LFBs model), obtaining wider working range than by chemiluminiscence measurements and good values of sensitivity and limit of detection. The main drawback of Inoue et al. (2007)'s method is that the electrochemical assay is performed separately. As the reduction is carried out as an additional step, the electrodes are not truly integrated into the LFS and it has to be cut. Some later designs (Wang et al., 2011b; Du et al., 2012) tried to fuse the electrode and the strip inside a cassette, including a cutter to section/cut the TL once the immunoassay was completed. 


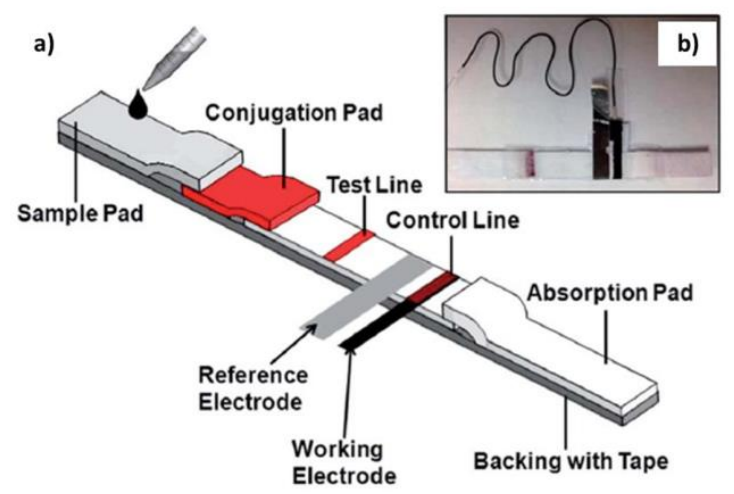

Fig. 13 a) Schematic representation of a LFS with electrical chip coupled and b) its corresponding photography. Reprinted with permission from Zhu, X.; Shah, P.; Stoff, S.; Liu, H.; Li, C. Z., 2014, Analyst, 139, 2850-2857. Copyright 2014 Royal Society of Chemistry.

Liu et al. (2007), Lin et al. (2008), Zhu et al. (2014); Akanda et al. (2014) integrated the electrodes in the LFS on different ways. On the cases of Liu et al. (2007) and Lin et al. (2008), QDs containing cadmium were used; after the test was performed and QDs were captured on TL, chlorhydric acid was used to release cadmium ions from QDs, which were detected by the electrodes, located under the $\mathrm{TL}$, when a detection solution $(\mathrm{Hg} / \mathrm{Bi})$ was added on it. The obtained limit of detection was really low, 30 and 20 $\mathrm{pg} / \mathrm{mL}$ respectively in each report. Interestingly, Liu et al. (2007) observed that when the immunoassay time was extended, the detection limit was decreased to $10 \mathrm{pg} / \mathrm{mL}$. In the work of Zhu et al. (2014) the colorimetric and electrochemical measurements were performed at the same time, without the addition of detection solution, thanks to the integration of commercial CNTs paper in the LFS as working electrode, placed on the CL, and printed silver/silver chloride ink as reference and counter electrode (Fig. 13a), being the electrodes laminated to ensure the contact between them and the nitrocellulose membrane (Fig. 13b). Following a competitive LFBs model and using AuNPs as labels, the colorimetric response was measured comparing the TL on photographs of the LFS and, at the same time, the chronoamperometric response was measured from the remaining AuNPs on the CL.

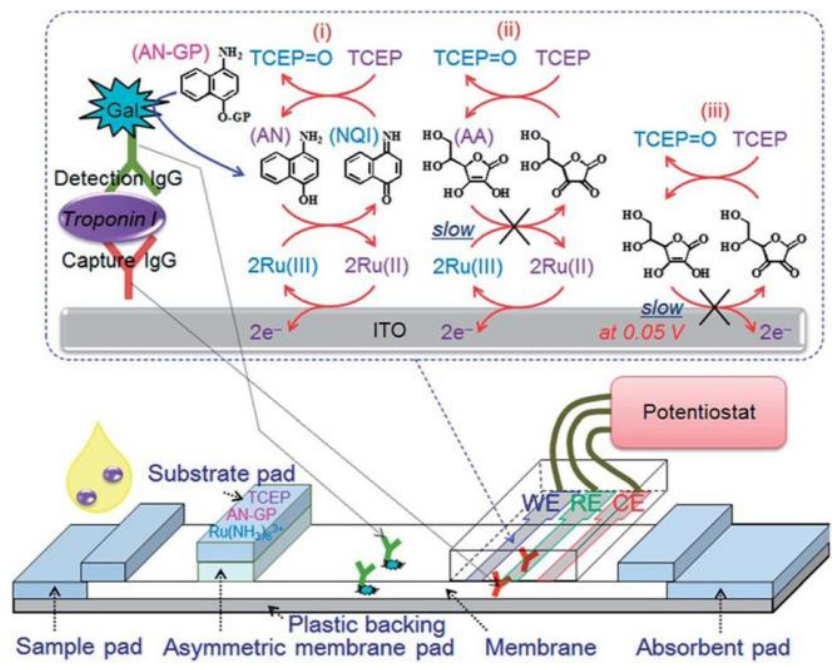

Fig. 14 Electrochemical immunoassay occurring on a LFS with an additional substrate pad. Reprinted with permission from Akanda, M. R.; Joung, H. A.; Tamilavan, V.; Park, S.; Kim, S.; Hyun, M. H.; Kim, M. G.; Yang, H., 2014, Analyst, 139, 1420. Copyright 2014 Royal Society of Chemistry.

It must be highlighted the work of Akanda et al. (2014), where they report a really low limit of detection, of only $0.1 \mathrm{pg} / \mathrm{mL}$. Their LFS includes an extra pad with different substrates to perform a redox reaction on the electrodes which allows detecting the analyte without being affected by electroactive interfering species present on the sample (Fig. 14). Thanks to this pad the need for sample pre-treating for both eliminating of interferences and to perform the electrochemical reaction is taken away, simplifying the test for the end user. On this design the electrodes, previously printed through photolithography on indium tin oxide substrate 
(ITO), were placed directly over the membrane, onto the detection antibodies. Hence, the detection on the LFS is only electrochemical and the strip does not provide optical checking.

\section{Other detections}

\subsection{Magnetic methods}

MNPs, as previously discussed, can be used as labels on lateral flow (Liu et al.; 2011) due to the fact they are strongly colored and can be easily conjugated with biomarkers. Usually, MNPs are used to preconcentrate the analyte in a sample through an washing step (Fisher et al., 2009; Liu et al., 2015), or are conjugated with colored particles (e.g. AuNPs) to assemble them, to help in the purification of the conjugate by means of magnetic separation and to increase the intensity of the color in TL (Tang et al., 2009). Nevertheless, MNPs can be used to a more interesting purpose; the magnetic field that the nanoparticles generate can be measured through a proper magnetic reader and transformed into a useful analytical signal. The advantage of this technique is that all the nanoparticles in the detection line should produce signal, contrary to optical and electrochemical methods where only nanoparticles on the surface or in contact with the electrodes, respectively, contribute significantly on the signaling.
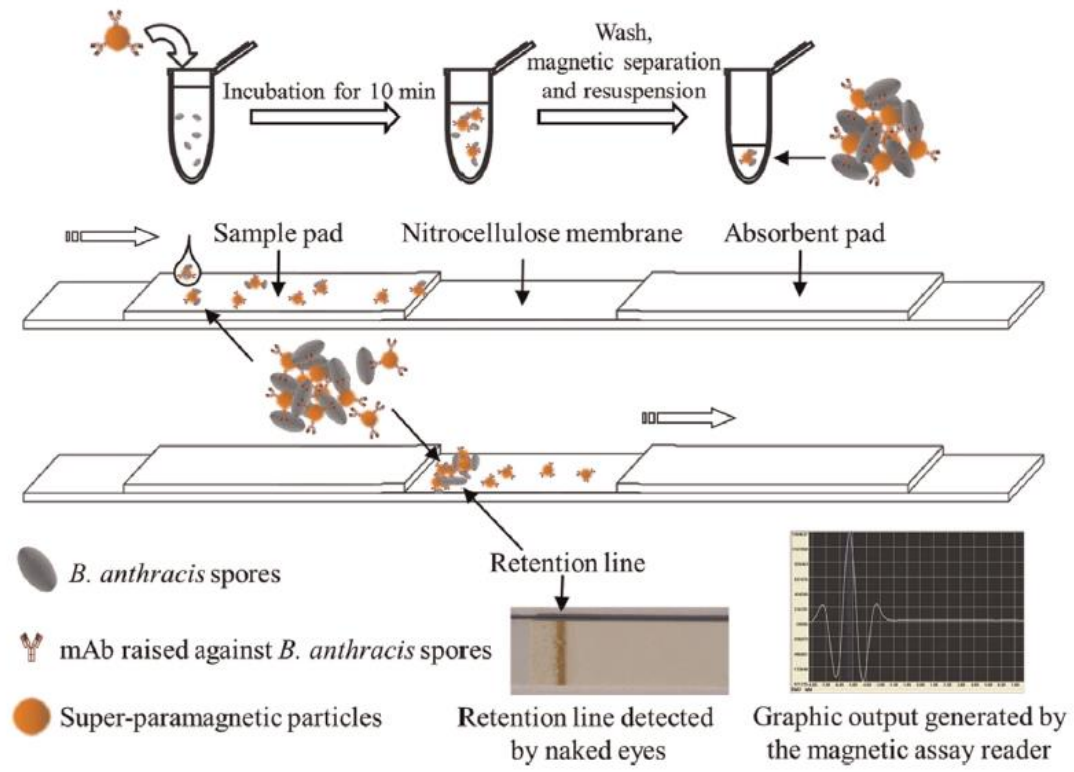

Fig. 15. LFS developed with MNPs as labels but without antibodies on the detection pad; "road closure effect". Reprinted with permission from Wang, D. B., Tian, B., Zhang, Z. P.; Wang, X. Y.; Fleming, J.; Bi, L. J.; Yang, R. F.; Zhang, X. E., 2015, Biosens. Bioelectron., 67, 608-614. Copyright 2015 Elsevier.

There exist several methods to produce and measure a signal using MNPs, being commercial magnetic assay readers (MARs) the most popular. MARs generate a magnetic field which excites the MNPs that simultaneously will produce their own magnetic field, detectable using the sensing coils of the apparatus. This effect can qualitatively measure groups of MNPs. Wang et al. (2009), Zheng et al. (2012), Wang et al. (2013), Shi et al. (2015b), Barnett et al. (2014) and Wang et al. (2015) reported the use of MAR on their works, obtaining wide working ranges, high selectivity, robust assays, good sensitivity and lower limits of detection than standard AuNPs LFs. However, the intensity of the signal was related to the size of the MNPs, being the larger ones which produced higher responses being this a drawback inasmuch as it slows significantly the assay time. Another manner to increase the signal, producing a higher magnetic field, is to increase the concentration of magnetite inside MNPs. It must not be forgotten that MNPs are strongly colored, so colorimetric response could also be measured from these LFs.

Even though nitrocellulose membranes contain pores with widths of some micrometers, the largest MNPs, combined with big analytes as spores, bacteria or cells, could clog the pores obstructing the flow of the conjugates across the membrane producing retention lines ("road closure effect"). Curiously, Wang et al. (2015) took advantage of this inconvenience to develop a novel LFs free of dispensed antibodies on the detection pad. Merely, in the presence of analyte, the MNPs-analyte conjugate formed the retention line at the beginning of the nitrocellulose membrane and it was measured with the MAR (Fig. 15). A clear advantage of this design is the cheapness of the LFSs, due to the fact that antibodies printed on it use to represent near the half of its costs; also the assay is faster than in the other magnetic LFs; nonetheless the absence of CL is a huge drawback because it impedes the detection of false positives.

In addition to MAR, other technologies like the use of giant magnetoresistive sensors (GMR) are emerging (Taton et al., 2009; Ryu et al., 2011; Marquina et al., 2012), GMR signaling is produced when the electrical resistance of the sensor is reduced by the 
effect of an external magnetic field derived from the MNPs. The technique can show a quite low limit of detection (12 pg/mL, Taton et al., 2009) but seems that its robustness and sensitivity still have to be improved.
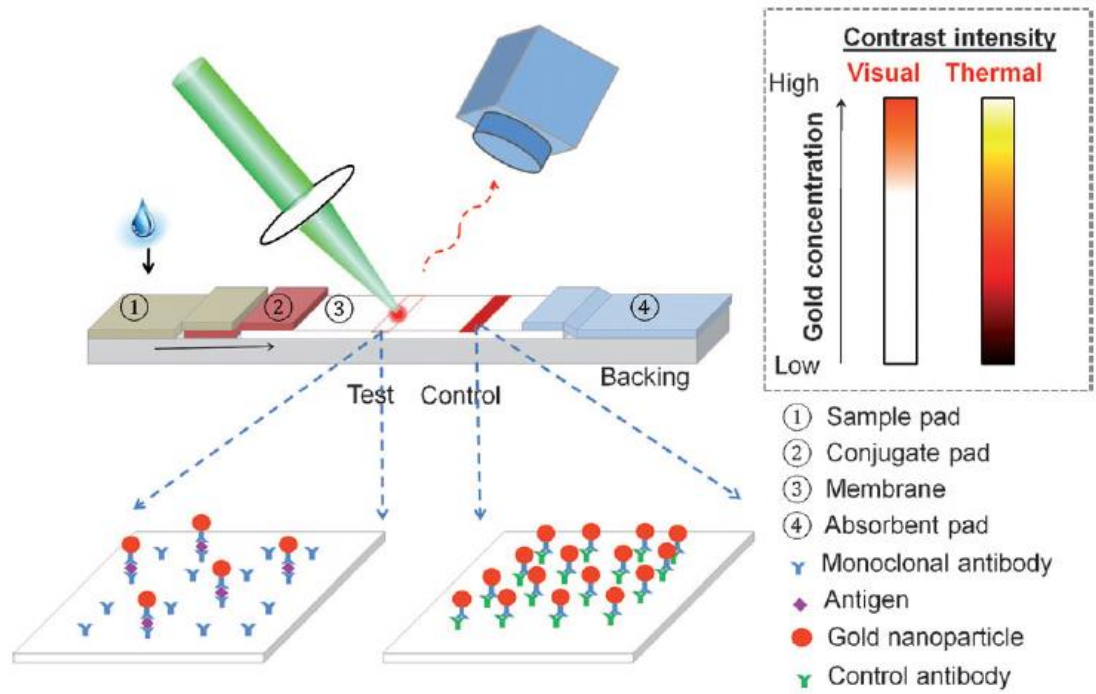

Fig. 16. a) Thermal Contrast Sensor applied on LFS. Reprinted with permission from Qin, Z.; Chan, W. C. W.; Boulware, D. R.; Akkin, T.; Butler, E. K.; Bischof, J. C., 2012, Angew. Chem. Int. Ed., 51, 4358-4361. Copyright 2012 Wiley-VCH.

\subsection{Other methods}

Thermal contrast is a technique that measures the radiation that the materials, over to absolute zero temperature, produce at infrared range. In LFS, thermal contrast can detect small variations of concentration which are not detectable with visible light, providing lower limits of detection than in the optical methods, including fluorescence in comparison to which, furthermore, avoids photobleaching effect; for this reason thermal contrast is considered a really robust and reproducible technique. Qin et al. (2012) reported the use of thermal contrast sensor (Fig. 16) on AuNPs LFS and compared the registered data against visual detection, reaching a 32-fold improvement on the sensitivity. Besides spherical nanoparticles, other shapes were tested (nanorods and nanoshells), by becoming shell AuNPs the one which exhibited higher response on thermal contrast.

A technique which can be combined with LFBs to enhance its response is isotachophoresis, an electrophoretic technique used to displace and preconcentrate several kinds of compounds, from large biocompounds to small inorganic ions, forcing their displacement with other ions. Moghadam et al. (2014, 2015) used this technique on LFSs to concentrate the conjugate and to transport it to the TL. The time of the assay is reduced, as well as the limit of detection, however must be taken into account that this method requires calibration, membrane pretreatment and current application (in which is strongly dependent the sensitivity of the device).

Another detection method which can be applied on LFS is surface-enhanced Raman scattering (SERS), a technique that enhances the Raman scattering of molecules (Raman reporters: chromophores or fluorescent dyes with high photostability and that exhibit resonance at Raman frequencies) previously attached in the label nanoparticles, increasing significantly the sensitivity of the assay. Li et al. (2014) reported the use of LFSs on SERS, using AuNPs covered with 4-mercaptobenzoic acid (a Raman reporter). Their experiments demonstrated the great precision of the technology, the high selectivity against the reporters and a really low limit of detection, $0.32 \mathrm{pg} / \mathrm{mL}$. 


\section{Integration and connection with real world applications}

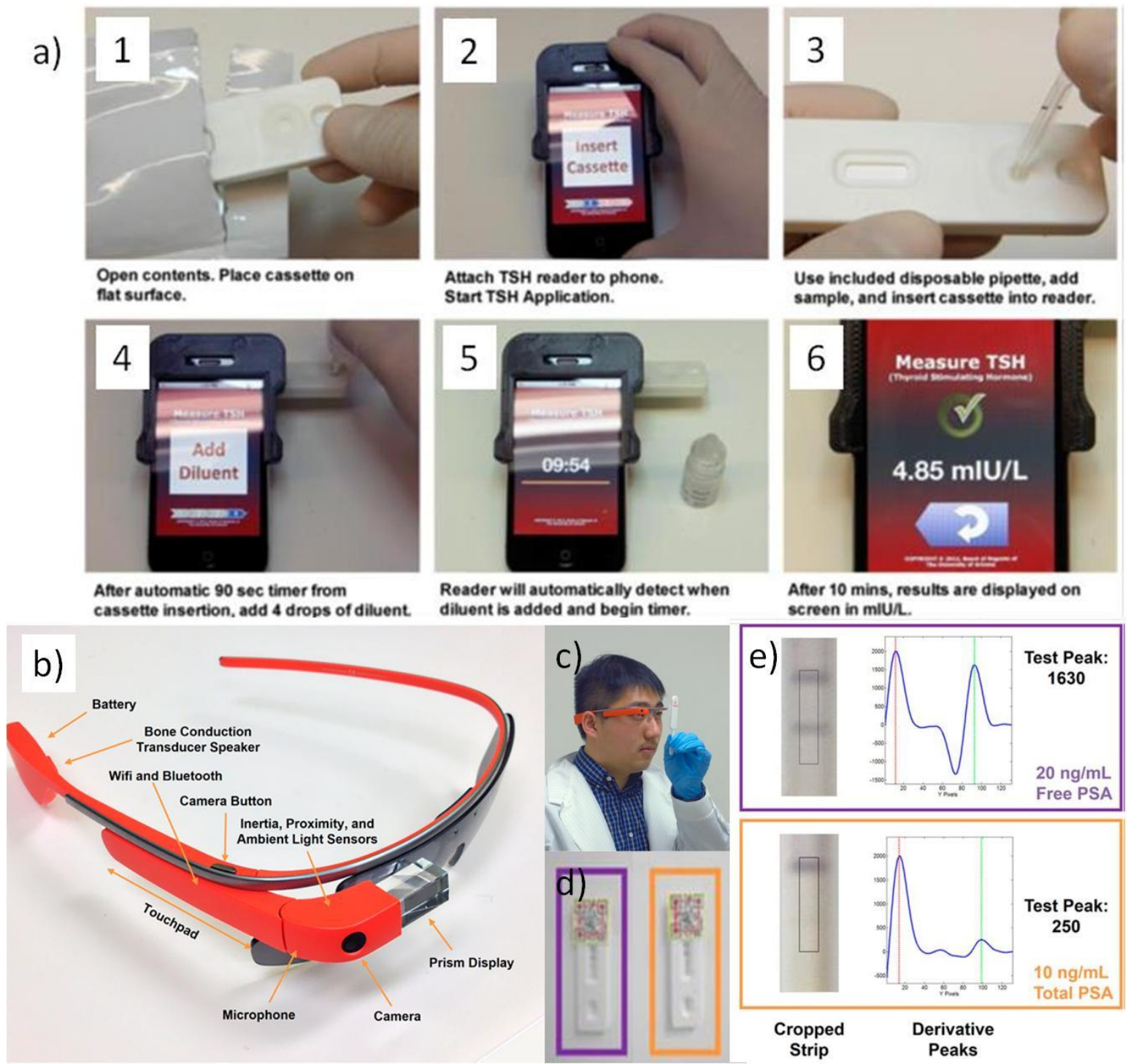

Fig. 17. a) Performance of a lateral flow assay assisted with a smartphone. Smartphone is used as a LFS reader. Adapted with permission from You, D. J.; Park, T. S.; Yoon, J. Y., 2013, Biosens. Bioelectron., 40, 180-185. Copyright 2013 Elsevier. b) Google glasses, used to read LFS (c) identified with QR codes (d) and the response obtained (e). Adapted with permission from Feng, S.; Caire, R; Cortazar, B.; Turan, M.; Wong, A.; Ozcan, A., 2014, ACS Nano, 8(3), 3069-3079. Copyright 2014 American Chemical Society.

The use of LFBs is not restricted only to laboratory or hospital environments. LFBs are designed to be portable devices which can be used overall at home or in the field. Their use is expected to be increased in the future so, consequently, it is important to adapt LFBs to the end user. At that point, it is necessary to focus not only in the LFS design, but also to the reader that should be user-friendly and low-cost. For example, Gui et al. (2014) demonstrated that with a charge-coupled device-based reader, which 
works on a similar way as a bar-code reader (in addition to some algorithms) a LFS developed with QDs (fluorescent measurements) could be easily read with low noise and low detection limits $(20 \mathrm{pg} / \mathrm{mL})$.

Nowadays, smartphones have become an almost indispensable tool for day to day life, with a countless number of applications and utilities; therefore, its connection with biosensing seems to be expectable. Mudanyali et al. (2012), You et al. (2014) and Zangheri et al. (2015) developed different user-friendly apps for smartphones which, with their respective adapter (containing LEDsource of light, filtering lens and a space to introduce LFS), can be used to detect the TL and CL of LFSs and translate the signal meanwhile all the steps that the user should perform during the assay are explained on the smartphone's screen (Fig. 17a).

With the arrival of the new Google Glasses (Fig. 17b) and similar products to the market the integration of augmented reality apps in real life will start. Feng et al. (2014) developed a voice-controlled app for Google Glasses that allows, by looking with your own eyes a QR code (quick response code) (Fig. 17c,d) printed in the LFS, get a quantitative interpretation of what your naked-eyes are watching (Fig. 17e). When looking to the QR, the Google Glasses get access into a server where a LFS picture will be saved in a database, analyzed and translated, receiving a posteriori the data on Google Glasses display or checking them in internet browser.

\section{Conclusions and future perspectives}

Table 1. Comparison of most remarkable methods and materials used in lateral flow biosensors.

\begin{tabular}{|c|c|c|c|}
\hline Detection method & Labels & Advantages & Disadvantages \\
\hline Optical (colorimetry) & & $\begin{array}{l}\text { - Naked-eye detection } \\
\text { - Fast qualitative response }\end{array}$ & $\begin{array}{l}\text { - Normally, only qualitative or } \\
\text { semiquantitative response }\end{array}$ \\
\hline & AuNPs & $\begin{array}{l}\text { - Easy to synthetize and modify } \\
\text { - Highly biocompatible and } \\
\text { versatile } \\
\text { - Intense colored } \\
\text { - Relatively inexpensive }\end{array}$ & $\begin{array}{l}\text { - Without the application of } \\
\text { enhancement techniques, poor } \\
\text { sensitivity and limits of detection } \\
\text { in comparison to other methods. }\end{array}$ \\
\hline & Carbon based materials & $\begin{array}{l}\text { - Strong contrast against } \\
\text { background } \\
\text { - Different shapes and behaviors } \\
\text { - Inexpensive and stable-in-time }\end{array}$ & $\begin{array}{l}\text { - Unspecific adsorptions } \\
\text { - Some forms have big-size: slow } \\
\text { response assay }\end{array}$ \\
\hline & $\begin{array}{l}\text { Nano/micro particles } \\
\text { loaded/modified with dyes }\end{array}$ & $\begin{array}{l}\text { - Inexpensive and high variety } \\
\text { of commercial products } \\
\text { - Good sensitivity and limits of } \\
\text { detection }\end{array}$ & $\begin{array}{l}\text { - Difficult to synthetize and modify } \\
\text { - Require to load high quantities of } \\
\text { dye to have a good signaling }\end{array}$ \\
\hline \multirow[t]{3}{*}{ Optical (fluorescence) } & & $\begin{array}{l}\text { - High sensitivity } \\
\text { - Low limits of detection }\end{array}$ & $\begin{array}{l}\text { - Naked- eye detection normally is } \\
\text { not possible } \\
\text { - Requires equipment with both } \\
\text { excitation light and fluorescence } \\
\text { measurement } \\
\text { - Materials normally exhibit } \\
\text { photobleaching effect, lose } \\
\text { intensity in time }\end{array}$ \\
\hline & QDs & $\begin{array}{l}\text { - Small-sized: fast assay } \\
\text { - Strong intensity }\end{array}$ & $\begin{array}{l}\text { - Difficult to synthetize and } \\
\text { conjugate }\end{array}$ \\
\hline & $\begin{array}{l}\text { Other fluorescent materials (ex. } \\
\text { UPTs) }\end{array}$ & $\begin{array}{l}\text { - Could require less energy to be } \\
\text { excited than QDs }\end{array}$ & $\begin{array}{l}\text { - Rare elements can be expensive } \\
\text { - Big-sized: slow assay }\end{array}$ \\
\hline Electrochemical & Electroactive nanoparticles & $\begin{array}{l}\text { - Highly sensitive } \\
\text { - Low limits of detection } \\
\text { - Devices easily miniaturized } \\
\text { and cheap }\end{array}$ & $\begin{array}{l}\text { - Requires equipment to produce } \\
\text { and translate the signal } \\
\text { - Reproducibility problems related } \\
\text { to electrodes }\end{array}$ \\
\hline Magnetic & $\begin{array}{l}\text { Nano/micro particles } \\
\text { loaded/modified with magnetite }\end{array}$ & $\begin{array}{l}\text { - Relatively inexpensive } \\
\text { - Colorimetric response is also } \\
\text { possible (multi signals) }\end{array}$ & $\begin{array}{l}\text { - Require magnetic detectors } \\
\text { - Sensitivity related to the size of } \\
\text { the particles: slow assays }\end{array}$ \\
\hline
\end{tabular}




\begin{tabular}{|c|c|c|c|}
\hline Thermal contrast & AuNPs & $\begin{array}{l}\text { - High improvement of } \\
\text { sensitivity and reproducibility } \\
\text { - Any material can emit signal }\end{array}$ & - Expensive equipment \\
\hline SERS & Raman reporter materials & $\begin{array}{l}\text { - High improvement of } \\
\text { sensitivity and detection limits }\end{array}$ & $\begin{array}{l}\text { - Expensive equipment } \\
\text { - Requires pretreatment of the } \\
\text { nanomaterials }\end{array}$ \\
\hline
\end{tabular}

LFSs have demonstrated to be affordable and portable tools to detect a huge variety of compounds (e.g. proteins, metallic ions, organic molecules, DNA, cells, etc.) in a really short period of time usually shorter than 30 min that may include pre- and posttreatments if necessary. As shown in table 1, LFSs are compatible with different detection methods and nanomaterials in which strongly depend the costs, the robustness, the sensitivity and the detection limits of these devices. The variety of properties of nanoparticles allows the secondary reactions to be performed onto their surface or their assembly and other modifications enhancing the intensity of the analytical signals. However, the signal of a LFS can also be easily enhanced just by modifying its architecture allowing tuning of the microfluidics beside other changes.

The progress in the LFB field is strongly related with the development of new nanomaterials, strategies and methods to increase the sensitivity and decrease the limit of detection. To reach that goal various strategies may be followed: a) use of nanoparticle labels which are simple to detect, stable and ensure intense signals; b) development of strategies that increase the quantity of signaling labels per unit of analyte; c) application of detection methods that inherently provide strong signaling and a high level of quantification in the detection zone; d) combination of different detection techniques and enhancement strategies already reported could help to offset the drawbacks of each system. The increase recently of the research in the field of graphene may offer new opportunities also for LFB technology. The special properties of graphene oxide in various optical biosensing technologies (Morales-Narváez and Merkoçi, 2012) including its quenching properties on QDs (Morales-Narváez et al., 2012), if applied on LFSs, may bring interesting results in terms of sensitivity and limit of detection.

LFSs are expected to deepen more in fast diagnostics, enabling its use at home to identify diseases or allergens, or simply to watch over on biological parameters that affect our security and health; because of this, electronic devices of common use, like smartphones or augmented reality apparatus (like the new Google Glasses) or future technologies, may probably be able to work as readers of LFSs, providing comprehensible test instructions and results to the user.

\section{Acknowledgements}

The authors acknowledge support from the Severo Ochoa Program (MINECO, Grant SEV-2013-0295).

\section{References}

Abera, A., Choi, J.W., 2010. Anal. Methods 2, 1819-1822.

Ahmad, A.L., Low, S.C., Shukor, S.R.A., Ismail, A., 2009. Ind. Eng. Chem. Res. 48, 3417-3424.

Akanda, M.R., Joung, H.A., Tamilavan, V., Park, S., Kim, S., Hyun, M.H., Kim, M.G., Yang, H., 2014. Analyst $139,1420$.

Anfossi, L., Giovannoli, C., Giraudi, G., Biagioli, F., Passini, C., Baggiani, C., 2012. J. Agric. Food Chem. 60, $11491-11497$.

Anfossi, L., Nardo, F.D., Giovannoti, C., Passini, C., Baggiani, C., 2013. Anal. Bioanal. Chem. 405, 9859-9867.

Aragay, G., Pons, J., Merkoçi, A., 2011. Chemical Reviews 111(5), 3433-3458.

Aragay, G., Pino, F., Merkoçi, A., 2012. Chemical Reviews 112(10), 5317-5338.

Assadollahi, S., Reininger, C., Palkovits, R., Pointl, P., Schalkhammer, T., 2009. Sensors 9, 6084-6100.

Baeumner, A.J., Jones, C., Wong, C.Y., Price, A., 2004a. Anal. Bioanal. Chem. 378, 1587-1593.

Baeumner, A.J., Pretz, J., Fang, S., 2004b. Anal. Chem. 76, 888-894.

Bai, Y., Tian, C., Wei, X., Wang, Y., Wang, D., Shi, X., 2012. RSC Advances 2, 1778-1781.

Barnett, J. M., Wraith, P., Kiely, J., Persad, R., Hurley, K., Hawkins, P., Luxton, R., 2014. Biosensors 4, $204-220$.

Berlina, A.N., Taranova, N.A., Zherdev, A.V., Vengerov, Y.Y., Dzantiev, B.B., 2013. Anal. Bioanal. Chem. 405, 4997-5000.

Blažková, M., Mičková-Holubová, B., Rauch, P., Fukal, L., 2009, Biosens. Bioelectron., 25, 753-758.

Blažková, M., Rauch, P., Fukal, L., 2010. Biosens. Bioelectron. 25, 2122-2128.

Bruno, J.G., 2014. Pathogens, 3, 341-355.

Campbell, K., Fodey, T., Flint, J., Danks, C., Danaher, M., O’Keeffe, M., Kennedy, D.G., Elliott, C., 2007. J. Agric. Food Chem. 55, 2497-2503.

Cazacu, A.C., Demmler, G.J., Neuman, M.A., Forbes, B.A., Chung, S., Greer, J., Alvarez, A.E., Williams, R., Bartholoma, N.Y., 2004. J. Clin. Microbio. 42(8), 3661-3664.

Chen, J., Fang, Z., Lie, P., Zeng, L., 2012. Anal. Chem. 84, 6321-6325.

Cho, J.H., Paek, S.H., 2001. Biotechnol. Bioeng. 75(6), 725-732.

Cho, J.H., Han, S.M., Paek, E.H., Cho, I.H., Paek, S.H., 2006. Anal. Chem. 78, 793-800.

Choi, D.H., Lee, S.K., Oh, Y.K., Bae, W.B., Lee, S.D., Kim, S., Shin, Y.B., Kim, M.G., 2010. Biosens, Bioelectron. 25, 19992002 . 
Choi, E.S., Lee, S.G., Lee, S.J., Kim, E., 2014. Biotechnol. Lett. 37, 627-632.

Clark Jr., L.C., Wolf, R., Granger, D., Taylor, Z., 1953. J. Appl. Physiol. 6(3), 189-93.

Cordray, M. S., Richards-Kortum, R. R., 2012, Am. J. Trop. Med. Hyg., 87(2), 223-230.

Corstjens, P.L.A.M., van Lieshout, L., Zuiderwijk, M., Kornelis, D., Tanke, H., Deelder, A.M., van Dam, G.J., 2008a. J. Clin. Microbiol. 46(1), 171-176.

Corstjens, P.L.A.M., Zuiderwijk, M., Brink, A., Li, S., Feindt, H., Niedbala, R. S., Tanke, H., 2001. Clin. Chem. 47(10), 18851893.

Corstjens, P.L.A.M., Zuiderwijk, M., Nilsson, M., Feindt, H., Niedbala, R.S., Tanke, H., 2003, Anal. Biochem. 312, $191-200$.

Corstjens, P.L.A.M., Zuiderwijk, M., Tanke, H., van der Ploeg-Schip, J., Ottenhoff, T.H.M., Geluk, A., 2008b. Clin Biochem. 41(6), 440-444.

Costa, M.N., Veigas, B., Jacob, J.M., Santos, D.S., Gomes, J., Baptista, P.V., Martins, R., Inácio, J., Fortunato, E., 2014. Nanotech. 25, 1-12.

De la Escosura-Muñiz, A., Parolo, C., Merloçi, A., 2010. Mater. Today 13, 24-34.

Du, D., Wang, J., Wang, L., Lu, D., Lin, Y., 2011. Anal. Chem. 84, 1380-1385.

Elenis, D.S., Ioannou, P.C., Christopoulos, T. K., 2011. Nanotechnology 22, 155501-155509.

Edwards, K.A., Baeumner, A.J., 2006. Anal. Bioanal. Chem. 386, 1335-1343.

Fang, Z., Huang, J., Lie, P., Xiao, Z., Ouyang, C., Wu, Q., Wu, Y., Liu G., Zeng, L., 2010. Chem. Commun. 46, $9043-9045$.

Feng, S., Caire, R, Cortazar, B., Turan, M., Wong, A., Ozcan, A., 2014. ACS Nano 8(3), 3069-3079.

Fenton, E.M., Mascarenas, M.R., López, G.P., Sibbett, S.S., 2009. ACS Appl. Mater. Inter. 1(1), 124-129.

Fisher, M., Atiya-Nasagi, Y., Simon, I., Gordin, M., Mechaly, A., Yitzhaki, S., 2009. Lett. Appl. Microbiol. 48, 413-418.

Fridley, G.E., Le, H.Q., Fu E., Yager, P., 2012. Lab Chip 12, 4321-4327.

Fu, E., Lutz, B., Kauffman, P., Yager, P., 2010. Lab Chip 10, 918-920.

Fu, E., Liang, T., Houghtaling, J., Ramachandran, S., Ramsey, S.A., Lutz, B., Yager, P., 2011a. Anal. Chem. 83, $7941-7946$.

Fu, E., Liang, T., Spicar-Mihalic, P., Houghtaling, J., Ramachandran, S., Yager, P., 2012. Anal Chem. 84(10), 4574-4579.

Fu, E., Ramsey, S.A., Kauffman, P, Lutz, B., Yager, P., 2011b. Microfluid. Nanofluid. 10, 29-35.

Ge, C., Yu, L., Fang, Z., Zeng, L., 2013. Anal. Chem. 85, 9343-9349.

Greenwald, R., Esfandiari, J., Lesellier, S., Houghton, R., Pollock, J., Aagaard, C., Andersene, P., Hewinson, R.G., Chambers,

M., Lyashchenko, K., 2003. Diagn. Micr. Infec. Dis. 46, 197-203.

Gui, C., Wang, K., Li, C., Dai, X., Cui, D., 2014. Nanoscale Res. Lett. 9(1), 57-64.

He, L., Nan, T., Cui, Y., Guo, S., Zhang, W., Zhang, R., Tan, G., Wang, B, Cui, L., 2014. Malar. J. 13, $127-136$.

He, Y., Zhang, S., Zhang, X., Baloda M., Gurung, A.S., Xu, H., Zhang, X., Liu, G., 2011. Biosens. Bioelectron. 26, $2018-2024$.

He, Y., Zeng, K., Zhang, S., Gurung, A.S., Baloda, M., Liu, G., 2012. Biosens. Bioelectron. 31(1), 310-315.

Held, J., Schmidt, T., Thornton, C.R., Kotter, E., Bertz, H., 2013. Infection 41(1), 1163-1169.

Ho, J.A., Wauchope, R.D., 2002. Anal. Chem. 74, 1493-1496.

Ho, J.A., Zeng, S.C., Tseng, W.H., Lin, Y.J., Chen, C.H., 2008. Anal. Bioanal. Chem. 391, 479-485.

Hong, S.Y., Park, Y.M., Jang, Y.H., Min, B.H., Yoon, H.C., 2012. BioChip J. 6(3), 213-220.

Hu, J., Wang, L., Li, F., Han, Y. L., Lin, M., Lu, T. J., Xu, F., 2013. Lab Chip, 13, 4352-4357.

Huang, X., Aguilar, Z.P., Li, H., Lai, W., Wei, H., Xu, H., Xiong, Y., 2013. Anal. Chem. 85, 5120-5128.

Huang, Y., Wen, W., Du, D., Zhang, X., Wang, S., Lin, Y., 2014. Biosens. Bioelectron. 61, 598-604.

Inoue, K., Ferrante, P., Hirano, Y., Yasukawa, T., Shiku, H., Matsue, T., 2007. Talanta 73, 886-892.

Jahanshahi-Anbuhi, S., Chavan, P., Sicard, C., Leung, V., Hossain, S.M.Z., Pelton, R., Brennan, J.D., Filipe, C.D.M., 2012. Lab

Chip 12, 5079-5085.

Kalogianni, D.P., Boutsika, L.M., Kouremenou, P.G., Christopoulos, T.K., Ioannou, P.C., 2011. Anal. Bioanal. Chem. 400, 11451152 .

Karakus, C., 2015. J. Immunoassay Immunochem. 36, 324-333.

Kersting, S., Rausch, V., Bier, F. F., Nickisch-Rosenegk, V., 2014. Malar. J. 13, 99-107.

Khreich, N., Lamourette, P., Boutal, H., Devilliers, K., Créminon, C., Volland, H., 2008. Anal. Biochem. 377, $182-188$.

Kim, K.Y., Shim, W.B., Kim, J.S., Chung, D.H., 2014. J. Food Sci. 79(10), 2048-2055.

Kim, Y.A., Lee, E.H., Kim, K.O., Lee, Y.T., Hammock, B.D., Lee, H.S., 2011. Anal. Chim. Acta 106-113.

Kim, Y.T., Chen, Y., Choi, J.Y., Kim, W.J., Dae, H.M., Jung, J., Seo, T.S., 2012. Biosens. Bioelectron. 33, 88-94.

Koets, M., Sander, I., Bogdanovic, J., Doekesc, G., van Amerongen, A., 2006. J. Environ. Monit. 8, 942-946.

Kolm, C., Mach, R.L., Krska, R., Brunner, K., 2015. Anal. Methods 7, 129-134.

Kuang, H., Xing, C., Hao, C., Liu, L., Wang, L., Xu, C., 2013. Sensors 13, 4214-4224.

Lee, J.Y., Kim, Y.A., Kim, M.Y., Lee, Y.T., Hammock, B.D., Lee, H.S., 2012. Anal. Chim. Acta 757, 69-74.

Leem, H., Shukla, S., Song, S., Heu, S., Kim, M., 2014. J. Food Safety 34, 239-248.

Li, C., Vandenberg, K., Prabhulkar, S., Zhu, X., Schneper, L., Methee, K., Rosser, C. J., Almeide, E., 2011. Biosens. Bioelectron. 26, 4342-4348.

Li, M., Yang, H., Li, S., Zhao, K., Li, J., Jiang, D., Sun, L., Deng, A., 2014. J. Agric. Food Chem. 62, 10896-10902. 
Li, X., Lu, D., Sheng, Z., Chen, K., Guo, X., Jin, M., Han, H., 2012. Talanta 100, 1-6.

Li, Z., Wang, Y., Wang, J., Tang, Z., Pounds, J.G., Lin, Y., 2010. Anal. Chem. 82, 7008-7014.

Lie, P., Liu, J., Fang, Z., Dun, B., Zeng, L., 2012. Chem. Commun. 48, 236-238.

Lin, Y.Y., Wang, J., Liu, G., Wu, H., Wai, C. M., Lin, Y., 2008. Biosens. Bioelectron. 23, 1659-1665.

Linares, E.M., Kubota, L.T., Michelis, J., Thalhammer, S., 2012. J. Immunol. Methods 375, 264-270.

Liu, B.H., Hung, C.T., Lu, C.C., Chou, H.N., Yu, F.Y., 2014. J. Agric. Food Chem. 62, 1254-1260.

Liu, C., Jia, Q., Yang, C., Qiao, R., Jing, L., Wang, L., Xu, C., Gao, M., 2011. Anal. Chem. 83, 6778-6784.

Liu, C., Ma, W., Gao, Z., Huang, J, Hou, Y., Xu, C., Yang, W., Gao, M., 2014. J. Mater. Chem. C 2, 9637-9642.

Liu, C., Qiu, X., Ongagna, S., Chen, D., Chen, Z., Abrams, W.R., Malamud, D., Corstjens, P.L.A.M., Bau, H. H., 2009. Lab Chip

9, 768-776.

Liu, D., Huang, Y., Wang, S., Liu, K., Chen, M., Xiong, Y., Yang, W., Lai, W., 2015. Food Control 51, $218-224$.

Liu, G., Lin, Y.Y., Wang, J., Wu, H., Wai, C. M., Lin, Y., 2007. Anal. Chem. 79, 7644-7653.

Liu, G., Mao, X., Phillips, J.A., Xu, H., Tan, W., Zeng, L., 2009. Anal. Chem. 81, 10013-10018.

Liu, J., Mazumdar, D., Lu, Y., 2006. Angew. Chem. Int. Ed. 45, 7955-7959.

Lönnberg, M., Carlsson, J., 2001. Anal. Biochem. 293, 224-231.

López-Marzo, A.M., Pons, J., Blake, A.D., Merkoçi, A., 2013a. Biosens. Bioelectron. 47, 190-198.

López-Marzo, A.M., Pons, J., Blake, A.D., Merkoçi, A., 2013b. Anal. Chem. 85, 3532-3538.

Lou, S.C., Patel, C., Ching, S.F., Gordon, J., 1993. J. Clin. Chem. 39(4), 619-624.

Lyashchenko, K.P., Greenwald, R., Esfandiari, J., Greenwald, D., Nacy, C.A., Gibson, S., Didier, P.J., Washington, M., Szczerba,

P., Motzel, S., Handt, L., Pollock, J.M., McNair, J., Andersen, P., Langermans, J.A.M., Verreck, F., Ervin, S., Ervin, F., McCombs,

C., 2007. Clin. Vaccine Immunol. 14(9), 1158-1164.

Mao, X., Ma, Y., Zhang, A., Zhang, L., Zeng, L., Liu, G., 2009. Anal. Chem. 81, 1660-1668.

Marquina, C., de Teresa, J.M., Serrate, D, Marzo, J., Cardoso, F.A., Saurel, D., Cardoso, S., Freitas, P.P., Ibarra, M.R., 2012. J.

Magn. Magn. Mater. 324, 3495-3498.

Mazumdar, D., Liu, J., Lu, G., Zhou, J., Lu, Y., 2010. Chem. Comm. 46,1416-1418.

Mei, Z., Qu, W., Deng, Y., Chu, H., Cao, J., Xue, F., Zheng, L., El-Nezamic, H.S., Wu, Y., Chen, W., 2013. Biosens. Bioelectron. 49, 457-461.

Merkoçi, A., 2010. Biosens. Bioelectron. 26, 1164-1177.

Moghadam, B.Y., Connelly, K.T., Posner, J.D., 2014. Anal. Chem. 86, 5829-5837.

Moghadam, B.Y., Connelly, K.T., Posner, J.D., 2015. Anal. Chem. 87, 1009-1017.

Morales-Narváez, E., Merkoçi, A., 2012. Adv. Mater. 24, 3298-3308.

Morales-Narváez, E., Pérez-López, B., Baptista Pires, L., Merkoçi, A., 2012. Carbon 50, 2987-2993.

Mudanyali, O., Dimitrov, S., Sikora, U., Padmanabhan, S., Navruza, I., Ozcan, A., 2012. Lab Chip 12, $2678-2686$.

Noguera, P., Posthuma-Trumpie, G.A., van Tuil, M., van der Wal, F.J., de Boer, A., Moers, A.P.H.A., van Amerongen, A., 2011.

Anal. Bioanal. Chem. 399, 831-838.

Nunes Pauli, G.E., de la Escosura-Muñiz, A., Parolo, C., Bechtold, I.H., Merkoçi, A., 2015. Lab Chip 15, $399-405$.

Oh, J.S., Ha, G.W., Cho, Y.S., Kim, M.J., An, D.J., Hwang, K.K., Lim, Y.K., Park, B.K., Kang, B.K., Song, D.S., 2006. Clin.

Vaccine Immunol. 13(4), 520-524.

Park, J.M., Jung, H.W., Chang, Y.W., Kim, H.S., Kang, M.J., Pyun, J.C., 2015. Anal. Chim. Acta 853, 360-367.

Parolo, C., Merkoçi, A., 2013. Che. Soc. Rev. 42, 450-457.

Parolo, C., de la Escosura-Muñiz, A., Polo, E., Grazú, V., de la Fuente, J.M., Merkoçi, A., 2013a. ACS Appl. Mater. Interfaces 5, 10753-10759.

Parolo, C., de la Escosura-Muñiz, A., Merkoçi, A., 2013b. Biosens. Bioelectron. 40, 412-416.

Parolo, C., Medina-Sánchez, M., de la Escosura-Muñiz, A., Merkoçi, A., 2013c. Lab Chip 13, 386-390.

Paterson, A.S., Raja, B., Garvey, G., Kolhatkar, A., Hagström, A.E.V., Kourentzi, K., Lee, T.R., Willson, R.C., 2014, Anal.

Chem. 86, 9481-9488.

Perfezou, M, Turner, A., Merkoçi, A., 2012. Chem. Soc. Rev. 41, 2606-2622.

Pesce, M. A., Chow, K.F., Hod, E., Spitalnik, S.L., 2006. Am. J. Clin. Pathol. 126(1), 61-70.

Preechakasedkit, P., Pinwattana, K., Dungchai, W., Siangproh, W., Chaicumpa, W., Tongtawe, P., Chailapakul, O., 2012.

Biosens. Bioelectron. 31, 562-566.

Qian, S., Bau, H.H., 2003. Anal. Biochem. 322, 89-98.

Qian, S., Bau, H.H., 2004. Anal. Biochem. 326, 211-224.

Qin, Z., Chan, W.C.W., Boulware, D.R., Akkin, T., Butler, E.K., Bischof, J.C., 2012. Angew. Chem. Int. Ed. 51, $4358-4361$.

Qiu, W., Xu, H., Takalkar, S., Gurung, A. S., Liu, B., Zheng, Y., Guo, Z., Baloda, M., Baryeh, K., Liu, G., 2015.

Bioelectron. 64, 367-372.

Rastogi, S.K., Gibson, C.M., Branen, J.R., Aston, D.E., Branen, A.E., Hrdlicka, P.J., 2012. Chem. Commun. 48, 7714-7716.

Rayev, M., Shmagel, K., 2008. J. Immunol. Methods 336, 9-15. 
Ren, M., Xu, H., Huang, X., Kuang, M., Xiong, Y., Xu, H., Xu, Y., Chen, H., Wang, A., 2014. ACS Appl. Mater. Interfaces 6, $14215-14222$

Renault, C., Koehne, J., Ricco, A.J., Crooks, R.M., 2014. Langmuir 30, 7030-7036.

Rivas, L., Medina-Sánchez, M., de la Escosura-Muñiz, A., Merkoçi, A., 2014. Lab Chip 14, 4406-4414.

Rohrman, B. R., Leautaud, V., Molyneux, E., Richards-Kortum, R.R., 2012. PLoS One 7(9), 45611-45618.

Rundström, G., Jonsson, A., Mårtensson, O., Mendel-Hartvig, I., Venge, P., 2007. Clin. Chem 53(2), 342-348.

Ryu, Y., Jin, Z., Kang, M.S., Kim, H.S., 2011. BioChip J. 5(3), 193-198.

Shi, C.Y., Deng, N., Liang, J.J., Zhou, K.N., Fu, Q.Q., Tang, Y., 2015a. Anal. Chim. Acta 854, 202-208.

Shi, L., Wu, F., Wen, Y., Zhao, F., Xiang, J., Ma, L., 2015b. Anal. Bioanal. Chem. 407(2), 529-535.

Shukla, S., Leem, H., Kim, M., 2011. Anal. Bioanal. Chem. 401, 2581-2590.

Shyu, R.H., Shyu, H.F., Liu, H.W., Tang, S. S., 2002. Toxicon 40, 255-258.

Song, S., Liu, N., Zhao, Z., Ediage, E.N., Wu, S., Sun, C., Saeger, S.D., Wu, A., 2014. Anal. Chem. 86, $4995-5001$.

Tang, D., Sauceda, J.C., Lin, Z., Ott, S., Basova, E., Goryacheva, I., Biselli, S., Lin, J., Niessner, R., Knopp, D., 2009. Biosens.

Bioelectron. 25, 514-518.

Tang, X., Zhang, Z., Li, P., Zhang, Q., Jiang, J., Wang, D., Lei, J., 2015. RSC Adv. 5, 558-564.

Taton, T., Johnson, D., Guire, P., Lange, E., Tondra, M., 2009. J. Magn. Magn. Mater. 321, 1679-1682.

Torabi, S.F., Lu, Y., 2011. Faraday Discuss. 149,125-135.

Van Dam, G.J., Wichers, J.H., Falcao Ferreira, T.M., Ghati, D., van Amerongen, A., Deelder, A.M., 2004. J. Clin. Microb. 42(12), 5458-5461.

Walcarius, A., Minteer, S.D., Wang, J., Lin, Y., Merkoçi, A., 2013. J. Mater. Chem. B 1, 4878-4908.

Wang, D.B., Tian, B., Zhang, Z.P., Deng, J.Y., Cui, Z.Q., Yang, R.F., Wang, X.Y., Wei, H.P., Zhang, X.E., 2013. Biosens.

Bioelectron. 42, 661-667.

Wang, D.B., Tian, B., Zhang, Z.P., Wang, X.Y., Fleming, J., Bi, L. J., Yang, R.F., Zhang, X.E., 2015. Biosens. Bioelectron. 67, 608-614.

Wang, L., Cai, J., Wang, Y., Fang, Q., Wang, S., Cheng, Q., Du, D., Lin, Y., Liu, F., 2014a. Microchim. Acta 181, $1565-1572$.

Wang, L., Chen, W., Ma, W., Liu, L., Ma, W., Zhao, Y., Zhu, Y., Xu, L., Kuang, H., Xu, C., 2011a. Chem. Commun. 47, 15741576 .

Wang, L., Lu, D., Wang, J., Du, D., Zou, Z., Wang, H., Smith, J.N., Timchalk, C., Liu, F., Lin, Y., 2011b. Biosens. Bioelectron. 26, 2835-2840.

Wang, X., Li, K., Shi, D., Xiong, N., Jin, X., Yi, J., Bi, D., 2007. J. Agric. Food Chem. 55, 2072-2078.

Wang, Y., Xu, Y., Wei, M., Gu, H., Xu, Q., Zhu, W., 2009. Mat. Sci. Eng. C 29, 714-718.

Wang, Y.K., Yan, Y. X., Ji, W.H., Wang, H., Li, S. Q., Zou, Q., Sun, J.H., 2013. J. Agric. Food Chem. 61, 5031-5036.

Wang, Z., Li, H., Li, C., Yu, Q., Shen, J., de Saeger, S., 2014b. J. Agric. Food Chem. 62, 6294-6298.

Wang, Z., Zhi, D., Zhao, Y., Zhang, H., Wang, X., Ru, Y., Li, H., 2014c. Int. J. Nanomedicine 9, 1699-1707.

Waters, W.R., Palmer, M.V., Thacker, T.C., Bannantine, J.P., Vordermeier, H.M., Hewinson, R.G., Greenwald, R., Esfandiari, J., McNair, J., Pollock, J.M., Andersen, P., Lyashchenko, K.P., 2006. Clin. Vaccine Immunol. 13(6), 648-654.

Wen, H.W., Borejsza-Wysocki, W., DeCory, T.R., Durst, R.A., 2005. Anal. Bioanal. Chem. 382, 1217-1226

Xia, X., Xu, Y., Zhao, X., Li, Q., 2009. Clin. Chem. 55, 1179-182.

Xie, Q.Y., Wu, Y.H., Xiong, Q.R., Xu, H.Y., Xiong, Y.H., Liu, K., Jin, Y., Lai, W.H., 2014. Biosens. Bioelectron. 54, $262-265$.

Xu, H., Chen, J., Birrenkott, J., Zhao, J.X., Takalkar, S., Baryeh, K., Liu, G., 2014. Anal. Chem. 86, 7351-7359.

Xu, H., Mao, X., Zeng, Q., Wang, S., Kawde, A.N., Liu, G., 2009. Anal. Chem. 81, 669-675.

Yang, H., Li, D., He, R., Guo, Q., Wang, K., Zhang, X., Huang, P., Cui, D., 2010. Nanoscale Res. Lett. 5, 875-881.

Yen, C.W., de Puig, H., Tam, J.O., Gómez-Márquez, J., Bosch, I., Hamad-Schifferli, K., Gehrke, L., 2015. Lab Chip 15, 16381641.

You, D.J., Park, T.S., Yoon, J.Y., 2013. Biosens. Bioelectron. 40, 180-185.

Zangheri, M., Cevenini, L., Anfossi, L., Baggiani, C., Simoni, P., Di Nardo, F., Roda, A., 2015. Biosens. Bioelectron. 64, 63-68. Zhang, F., Zou, M., Chen, Y., Li, J., Wang, Y., Qi, X., Xue, Q., 2014. Biosens. Bioelectron. 51, 29-35.

Zheng, C., Wang, X., Lu, Y., Liu, Y., 2012. Food Control 26, 446-452.

Zhou, J., Zhu, K., Xu, F., Wang, W., Jiang, H., Wang, Z., Ding, S., 2014. J. Agric. Food Chem. 62, 12061-12066.

Zhou, P., Lu, Y., Zhu, J., Hong, J., Li, B., Zhou, J., Gong, D., Montoya, A., 2004. J. Agric. Food Chem. 52, $4355-4359$.

Zhu, X., Shah, P., Stoff, S., Liu, H., Li, C. Z., 2014. Analyst 139, 2850-2857.

\section{Abbreviations}

AEC, 3-amino-9-ethylcarbazole; AuNPs, gold nanoparticles; CL, control line; CNPs, carbon nanoparticles; CNTs, carbon nanotubes; GMR, giant magnetoresistive sensor; HRP, horseradish peroxidase; IAs, immunochromatographic assays; LBs, latex beads; LFBs, lateral flow biosensors; LFS, lateral flow strip; MAR, magnetic assay readers; MNPs, magnetite nanoparticles; OVA, 
ovalbumin; TL, test line; TMB, 3,3',5,5'-tetramethylbenzidine; QDs, quantum dots; QR, quick response code; SERS, surfaceenhanced Raman scattering; UPTs, up-converting phosphor technologies. 